\title{
Phase transition in the massive Gross-Neveu model in toroidal topologies
}

\author{
F. C. Khanna, ${ }^{1,2, *}$ A. P. C. Malbouisson, ${ }^{3, \dagger}$ J. M. C. Malbouisson, ${ }^{4,5, \$}$ and A. E. Santana ${ }^{6, \S}$ \\ ${ }^{1}$ Theoretical Physics Institute, University of Alberta, Edmonton, Alberta T6G 2J1, Canada \\ ${ }^{2}$ TRIUMF, 4004, Westbrook Mall, Vancouver, British Columbia V6T 2A3, Canada \\ ${ }^{3}$ Centro Brasileiro de Pesquisas Físicas/MCT, 22290-180, Rio de Janeiro, RJ, Brazil \\ ${ }^{4}$ Instituto de Física, Universidade Federal da Bahia, 40210-340, Salvador, BA, Brazil \\ ${ }^{5}$ Departamento de Física, Faculdade de Ciências, Universidade do Porto, 4169-007, Porto, Portugal \\ ${ }^{6}$ Instituto de Física, International Center of Physics, Universidade de Brasília, 70910-900, Brasília, DF, Brazil
}

(Received 15 August 2011; published 11 April 2012)

\begin{abstract}
We use methods of quantum field theory in toroidal topologies to study the $N$-component $D$-dimensional massive Gross-Neveu model, at zero and finite temperature, with compactified spatial coordinates. We discuss the behavior of the large- $N$ coupling constant $(g)$, investigating its dependence on the compactification length $(L)$ and the temperature $(T)$. For all values of the fixed coupling constant $(\lambda)$, we find an asymptotic-freedom type of behavior, with $g \rightarrow 0$ as $L \rightarrow 0$ and/or $T \rightarrow \infty$. At $T=0$, and for $\lambda \geq \lambda_{c}^{(D)}$ (the strong-coupling regime), we show that, starting in the region of asymptotic freedom and increasing $L$, a divergence of $g$ appears at a finite value of $L$, signaling the existence of a phase transition with the system getting spatially confined. Such a spatial confinement is destroyed by raising the temperature. The confining length, $L_{c}^{(D)}$, and the deconfining temperature, $T_{d}^{(D)}$, are determined as functions of $\lambda$ and the mass $(m)$ of the fermions, in the case of $D=2,3,4$. Taking $m$ as the constituent quark mass $(\approx 350 \mathrm{MeV})$, the results obtained are of the same order of magnitude as the diameter $(\approx 1.7 \mathrm{fm})$ and the estimated deconfining temperature $(\approx 200 \mathrm{MeV})$ of hadrons.
\end{abstract}

DOI: 10.1103/PhysRevD.85.085015

PACS numbers: 11.30.Rd, 11.10.Wx

\section{INTRODUCTION}

The strong interaction among quarks and gluons, the constituents of the hadronic matter, has such a structure that obligates them to live spatially confined, at low temperatures, within distances $\sim 1 \mathrm{fm}$ in colorless states. It is usually accepted that in an earlier stage of the Universe, as it cooled down, quarks and gluons condensed into hadrons at an estimated temperature of the order of $200 \mathrm{MeV}$. At very high energies, deep inelastic scattering indicates that the quarks are nearly free, a regime denominated as asymptotic freedom.

In the standard model, the theory of strong interactions is quantum chomodynamics (QCD), which should describe such facts, accounting also for the nuclear forces. However, QCD has a very involved mathematical structure, which practically prevents us from finding analytical results taking into account both confinement and asymptotic freedom. Lattice calculations have been implemented to simulate the behavior of the theory in the confining region, both at zero and finite temperature, providing (among other results) an estimate of the deconfining temperature. Rigorous QCD calculations, both at zero and finite temperature, have been worked out $[1,2]$ but mainly treating the asymptotically free domain at high energies or high temperatures, where perturbation theory is applicable.

\footnotetext{
*fkhanna@ualberta.ca

†adolfo@cbpf.br

\#jmalboui@ufba.br

\$asantana@fis.unb.br
}

Because of the difficulty of treating QCD analytically, phenomenological approaches and studies of effective, simplified models have been stimulated along the years to give clues to the behavior of hadronic systems. A celebrated effective model, which shares with QCD some basic properties, is the Nambu-Jona-Lasinio (NJL) model [3]. One of its sectors, providing the simplest effective model which may be considered as describing quark interactions, is a direct four-fermion coupling, where gluon fields and color degrees of freedom are integrated out, resembling the Fermi treatment of the weak interaction. This corresponds to the Gross-Neveu (GN) model [4], considered in space-time dimension $D=4$. Although the GN model is not renormalizable for dimensions greater than $D=2$, the Euclidian model has been shown to exist and has been constructed for $D=3$ in the large- $N$ limit [5]. But, within the spirit of effective theories, perturbative renormalizability is not a requirement to have a physically meaningful model [6-10].

The GN model, as a prototype model for interacting fermions, has been analyzed extensively in recent years [11-20], including the study of continuous and discrete chiral symmetry [21,22]. For the version with $N$ massless fermions in $(2+1)$ dimensions, for instance, a chiral symmetry breaking is found in perturbative analysis, with the restoration of such a symmetry at finite temperature [23]. In particle physics, these results provide insights into the intricate structure of the hadronic matter, such as for the quark confinement/deconfinement phase transition [24,25]. 
The four-point contact interaction of the GN model is similar to the delta interaction in the BCS theory of superconductivity. In the latter case, as in other systems of condensed matter, the susceptibility arising from the linear response theory has a divergence at a finite temperature indicating the existence of a second-order phase transition between a disordered and a condensed phase [26]. Spontaneous symmetry breaking is the common feature underlying all these phenomena [27]. Recently [28], we have shown that such instability appears in the onecomponent massive tridimensional GN model at finite temperature. Here, we intend to use a similar treatment to investigate the existence of a phase transition in the massive, $N$-component, GN model.

We employ methods of quantum field theory in toroidal topologies [29-34] to extend previous results $[35,36]$ for the Euclidian massive, $N$-component, GN model in the large- $N$ limit. This amounts to considering the GN model in a $D$-dimensional space-time and to compactifying a $d$-dimensional $(d \leq D)$ subspace. The compactification is a generalization of the Matsubara procedure. The Matsubara imaginary-time formalism corresponds to considering fields in a space with topology $\mathbb{S}^{1} \times \mathbb{R}^{D-1}$, where $\mathbb{S}^{1}$ is a circumference of length $\beta$, with periodic (antiperiodic) boundary conditions for bosons (fermions). Such a compactification of the Euclidian time can be directly generalized to include the compactification of space coordinates as well. This allows us to consider field theoretical models with spatial constraints, at zero or finite temperature, by using generating functionals with a path-integral formalism on the topology $\mathbb{S}^{1_{1}} \times \cdots \times \mathbb{S}^{1_{d}} \times \mathbb{R}^{D-d}$ [29-32]. These ideas have been established recently on a firm foundation $[33,34]$ and applied in different physical situations, for example: for spontaneous symmetry breaking in the compactified $\phi^{4}$ model [37-39]; for second-order phase transitions in superconducting films, wires, and grains [40-42]; for the Casimir effect for bosons and fermions [43-48]; for size effects in the NJL model [49-53]; and for electrodynamics with an extra dimension [54].

We treat particularly the cases $D=2,3$, 4 with all spatial dimensions compactified, initially at zero temperature and then we discuss temperature effects by compactifying the imaginary time in a length $\beta=T^{-1}, T$ being the temperature. This corresponds to considering the system contained in a parallelepiped box, with antiperiodic boundary conditions on its faces, at finite $T$. We study the behavior of the system as a function of its size and of the temperature, concentrating on the dependence of the large- $N$ coupling constant on the compactification length and temperature. We show that, even at $T=0$, a singularity in the four-point function may appear driven by changes in the compactification length, suggesting the existence of a second-order phase transition in the system. This can be interpreted as a spatial confinement transition, which may be present in the massive version of the GN model.
We use concurrently dimensional and analytic regularizations and employ a subtraction scheme where the polar terms, arising from Epstein-Hurwitz generalized zetafunctions, are suppressed. Results obtained with this procedure have similar structure for all values of $D$, which gives us confidence that they are meaningful for the fourdimensional space-time. This is reinforced $a$ posteriori by the fact that the numerical results found for $D=4$ are of the same order of magnitude as the corresponding values for $D=2$ and $D=3$. For the massive GN model in the large- $N$ limit, discussed in the present paper, we obtain simultaneously an asymptotic-freedom type of behavior and spatial confinement, in the strong-coupling regime, for low temperatures. We also show that, as the temperature is increased, a deconfining transition occurs. We calculate the values of the confining lengths and the deconfining temperatures for $D=2,3,4$.

This article starts by discussing, in Sec. II, the $D$-dimensional massive, $N$-component, Gross-Neveu model with $d(\leq D)$ compactified dimensions. In Sec. III, the calculation of the effective large- $N$ coupling constant is carried out for the cases with $D=2,3,4$ at zero temperature. Temperature effects are presented in Sec. IV. The last section provides a comparison of our estimated confining lengths and deconfining temperatures with experimental values.

\section{COMPACTIFIED GROSS-NEVEU MODEL}

The massive GN model in a $D$-dimensional Euclidean space is described by the Wick-ordered Lagrangian density

$$
\mathcal{L}=: \bar{\psi}(x)(i \nabla+m) \psi(x):+\frac{u}{2}(: \bar{\psi}(x) \psi(x):)^{2},
$$

where $m$ is the mass, $u$ is the coupling constant, $x$ is a point of $\mathbb{R}^{D}$, and the $\gamma$ 's are the Dirac matrices. We consider the GN model in its $N$-component version, so that $\psi(x)$ represents a spin $\frac{1}{2}$ field having $N$ (flavor) components, $\psi^{a}(x)$, $a=1,2, \ldots, N$, with summations over flavor and spin indices being understood in Eq. (1). We calculate quantities of interest by taking the large- $N$ limit where $N \rightarrow \infty$ and $u \rightarrow 0$ in such way that $N u=\lambda$ remains finite. Throughout the text we use natural units with $\hbar=c=$ $k_{B}=1$.

Our main goal is to determine the large- $N$ (effective) coupling constant when $d(\leq D)$ Euclidian coordinates, say $x_{1}, \ldots, x_{d}$, are compactified, that is, considering the system in a topology $\mathbb{S}^{1_{1}} \times \cdots \mathbb{S}^{1} \times \mathbb{R}^{D-d}$. This corresponds to restricting the coordinates $x_{i}$ to segments of length $L_{i}(i=$ $1,2, \ldots d)$, with the fermionic field $\psi(x)$ satisfying antiperiodic boundary conditions. If all $x_{i}$ are spatial coordinates, the model refers to the system compactified in a $d$-dimensional box at zero temperature while, with one coordinate being the Euclidian time (say $x_{d}$ ), one has the system with $d-1$ compactified spatial dimensions at finite temperature; in this latter case, $L_{d}$ would stand for 
$\beta=1 / T$, the inverse of the temperature. For massless fermions, this spatial compactification with antiperiodic boundary conditions is equivalent to considering the system constrained to "live" inside a parallelepiped box, with edges $L_{i}(i=1,2, \ldots d)$, under bag model conditions (no outgoing currents) on parallel, opposite, faces [55,56]. In our case, to calculate $n$-point functions, we apply the generalized Matsubara prescription, which amounts to modifying the Feynman rules performing, the replacements

$$
\begin{array}{r}
k_{i} \rightarrow \nu_{i}=\frac{2 \pi\left(n_{i}+\frac{1}{2}\right)}{L_{i}}, \quad i=1,2, \ldots, d ; \\
\int \frac{d^{D} k}{(2 \pi)^{D}} F(k) \rightarrow \frac{1}{L_{1} \ldots L_{d}} \sum_{\left\{n_{i}\right\}=-\infty}^{+\infty} \int \frac{d^{D-d} \mathbf{k}}{(2 \pi)^{D-d}} F\left(\left\{k_{i}\right\}, \mathbf{k}\right),
\end{array}
$$

where $\left\{n_{i}\right\}=\left\{n_{1}, \ldots, n_{d}\right\}$, with $n_{i} \in \mathbb{Z}$, and $\mathbf{k}$ is a $(D-d)$-dimensional vector in momentum space; the discreet momenta $\nu_{i}$ are referred to as Matsubara frequencies. Additionally, it should be pointed out that the choice of antiperiodic boundary conditions for the spatial compactification, instead of the simpler periodic ones, is also due to the fact that they emerge naturally in the generalization of the Kubo-Martin-Schwinger (KMS) conditions satisfied by correlation functions for fermionic fields in toroidal topologies $[33,34]$.

We shall define the large- $N$ effective coupling constant between the fermions in terms of the four-point function at zero external momenta. The $\left\{L_{i}\right\}$-dependent four-point function, at leading order in $\frac{1}{N}$, is given by the sum of chains of one-loop (bubble) diagrams, which can be formally expressed as

$$
\Gamma_{D d}^{(4)}\left(0 ;\left\{L_{i}\right\}, u\right)=\frac{u}{1+N u \Pi_{D d}\left(\left\{L_{i}\right\}\right)},
$$

where the $\left\{L_{i}\right\}$-dependent one-loop Feynman diagram is given by

$$
\begin{aligned}
\Pi_{D d}\left(\left\{L_{i}\right\}\right)= & \frac{1}{L_{1} \cdots L_{d}} \sum_{\left\{n_{i}\right\}=-\infty}^{\infty} \int \frac{d^{D-d} \mathbf{k}}{(2 \pi)^{D-d}} \\
& \times\left[\frac{m^{2}-\mathbf{k}^{2}-\sum_{i=1}^{d} \nu_{i}^{2}}{\left(\mathbf{k}^{2}+\sum_{i=1}^{d} \nu_{i}^{2}+m^{2}\right)^{2}}\right] .
\end{aligned}
$$

Prior to defining an effective large- $N$ coupling constant, we have to deal with the ultraviolet divergences of $\Pi_{D d}\left(\left\{L_{i}\right\}\right)$. To simplify the use of regularization techniques, we introduce the dimensionless quantities $b_{i}=\left(m L_{i}\right)^{-2}(i=1, \ldots, d) \quad$ and $\quad q_{j}=k_{j} / 2 \pi m(j=$ $d+1, \ldots, D)$, in terms of which the one-loop diagram is written as

$$
\begin{aligned}
\Pi_{D d}\left(\left\{b_{i}\right\}\right) & \\
= & \left.\Pi_{D d}\left(s ;\left\{b_{i}\right\}\right)\right|_{s=2} \\
= & \frac{m^{D-2}}{4 \pi^{2}} \sqrt{b_{1} \cdots b_{d}}\left\{\frac{1}{2 \pi^{2}} U_{D d}\left(s ;\left\{b_{i}\right\}\right)\right. \\
& \left.\quad-U_{D d}\left(s-1 ;\left\{b_{i}\right\}\right)\right\}\left.\right|_{s=2},
\end{aligned}
$$

where

$$
\begin{aligned}
U_{D d} & \left(\mu ;\left\{b_{i}\right\}\right) \\
& =\sum_{\left\{n_{i}\right\}=-\infty}^{\infty} \int \frac{d^{D-d} \mathbf{q}}{\left[\mathbf{q}^{2}+\sum_{j=1}^{d} b_{j}\left(n_{j}+\frac{1}{2}\right)^{2}+(2 \pi)^{-2}\right]^{\mu}} .
\end{aligned}
$$

We find from Eq. (5) that $\Pi_{D d}$ has dimension of $m^{D-2}$, which is inverse of the mass dimension of the coupling constant.

We employ a modified minimal subtraction scheme which uses concurrently dimensional and analytical regularizations. In this scheme, the subtracted terms are poles (for even $D \geq 2$ ) of the Epstein-Hurwitz zeta-functions. First, using well-known dimensional regularization formulas to perform the integral over $\mathbf{q}=\left(q_{d+1}, \ldots, q_{D}\right)$ in Eq. (6), we obtain

$$
\begin{aligned}
U_{D d}\left(\mu ;\left\{b_{i}\right\}\right)= & \pi^{(D-d) / 2} \frac{\Gamma\left(\mu-\frac{D-d}{2}\right)}{\Gamma(\mu)} \sum_{\left\{n_{i}\right\}=-\infty}^{\infty}\left[\sum_{j=1}^{d} b_{j}\left(n_{j}+\frac{1}{2}\right)^{2}\right. \\
& \left.+(2 \pi)^{-2}\right]^{((D-d) / 2)-\mu} .
\end{aligned}
$$

The summations over half-integers in this expression can be transformed into sums over integers, leading to

$$
\begin{aligned}
U_{D d}\left(\mu ;\left\{b_{i}\right\}\right)= & \pi^{(D-d) / 2} \frac{\Gamma\left(\mu-\frac{(D-d)}{2}\right)}{\Gamma(\mu)} 4^{\eta} \\
& \times\left[Z_{d}^{h^{2}}\left(\eta, b_{1}, \ldots, b_{d}\right)-\sum_{i=1}^{d} Z_{d}^{h^{2}}\left(\eta, \ldots, 4 b_{i}, \ldots\right)\right. \\
& +\sum_{i<j=1}^{d} Z_{d}^{h^{2}}\left(\eta, \ldots, 4 b_{i}, \ldots, 4 b_{j}, \ldots\right)-\ldots \\
& \left.+(-1)^{d} Z_{d}^{h^{2}}\left(\eta, 4 b_{1}, \ldots, 4 b_{d}\right)\right]
\end{aligned}
$$

where $h^{2}=\pi^{-2}, \eta=\mu-\frac{D-d}{2}$, and

$$
Z_{d}^{h^{2}}\left(\eta,\left\{a_{i}\right\}\right)=\sum_{\left\{n_{i}\right\}=-\infty}^{\infty}\left[\sum_{j=1}^{d} a_{j} n_{j}^{2}+h^{2}\right]^{-\eta}
$$

is the multiple ( $d$-dimensional) Epstein-Hurwitz zetafunction.

The Epstein-Hurwitz zeta-function $Z_{d}^{h^{2}}\left(\eta,\left\{a_{i}\right\}\right)$ can be analytically extended to the whole complex $\eta$-plane [39], through a generalization of the procedure presented in Refs. [57,58]; then we find 


$$
\begin{aligned}
& Z_{d}^{h^{2}}\left(\eta,\left\{a_{i}\right\}\right)=\frac{\pi^{d / 2}}{\sqrt{a_{1} \cdots a_{d}} \Gamma(\eta)}\left[\frac{1}{h^{2(\eta-d)}} \Gamma\left(\eta-\frac{d}{2}\right)+\sum_{\theta=1}^{d} 2^{\theta+1} \sum_{\left\{\sigma_{\theta}\right\}\left\{n_{\sigma_{\theta}}\right\}=1}^{\infty}\left(\frac{\pi}{h} \sqrt{\frac{n_{\sigma_{1}}^{2}}{a_{\sigma_{1}}}+\cdots+\frac{n_{\sigma_{\theta}}^{2}}{a_{\sigma_{\theta}}}}\right)^{\eta-(d / 2)}\right. \\
& \left.\times K_{\eta-(d / 2)}\left(2 \pi h \sqrt{\frac{n_{\sigma_{1}}^{2}}{a_{\sigma_{1}}}+\cdots+\frac{n_{\sigma_{\theta}}^{2}}{a_{\sigma_{\theta}}}}\right)\right]
\end{aligned}
$$

where $\left\{\sigma_{\theta}\right\}$ represents the set of all combinations of the indices $\{1,2, \ldots, d\}$ with $\theta$ elements and $K_{\alpha}(z)$ is the Bessel function of the third kind. Consequently, the function $U_{D d}\left(\mu ;\left\{b_{i}\right\}\right)$ can also be analytically continued to the whole complex $\mu$-plane.

Taking $Z_{d}^{h^{2}}\left(\eta,\left\{a_{i}\right\}\right)$ given by Eq. (10), grouping similar terms appearing in the parcels of Eq. (8) and using the identity

$$
\sum_{j=1}^{N}\left(\frac{-1}{2}\right)^{j} \frac{N !}{j !(N-j) !}=\frac{1}{2^{N}}
$$

we obtain

$$
\begin{aligned}
U_{D d}\left(\mu ;\left\{b_{i}\right\}\right)= & \frac{2^{2 \mu-D} \pi^{2 \mu-(D / 2)}}{\Gamma(\mu)} \frac{1}{\sqrt{b_{1} \cdots b_{d}}} \\
& \times\left[\Gamma\left(\mu-\frac{D}{2}\right)+2^{D / 2} W_{D d}\left(\mu ;\left\{b_{i}\right\}\right)\right],
\end{aligned}
$$

with $W_{D d}\left(\mu ;\left\{b_{i}\right\}\right)$ given by

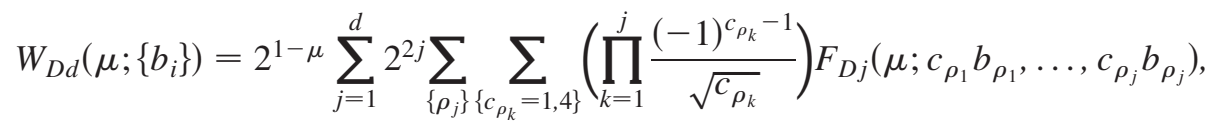

where $\left\{\rho_{j}\right\}$ stands for the set of all combinations of the indices $\{1,2, \ldots, d\}$ with $j$ elements and the functions $F_{D j}\left(\mu ; a_{1}, \ldots, a_{j}\right)$, for $j=1, \ldots, d$, are defined by

$$
\begin{aligned}
F_{D j}\left(\mu ; a_{1}, \ldots, a_{j}\right)= & \sum_{n_{1}, \ldots, n_{j}=1}^{\infty}\left(2 \sqrt{\frac{n_{1}^{2}}{a_{1}}+\cdots+\frac{n_{j}^{2}}{a_{j}}}\right)^{\mu-(D / 2)} \\
& \times K_{\mu-(D / 2)}\left(2 \sqrt{\frac{n_{1}^{2}}{a_{1}}+\cdots+\frac{n_{j}^{2}}{a_{j}}}\right) .
\end{aligned}
$$

Substituting Eq. (12) into Eq. (5) leads directly to an analytic extension of $\Pi_{D d}\left(s ;\left\{b_{i}\right\}\right)$ for complex values of $s$, in the vicinity of $s=2$. In fact, $\Pi_{D d}\left(s ;\left\{b_{i}\right\}\right)$ can be written as

$$
\begin{aligned}
\Pi_{D d}\left(s ;\left\{b_{i}\right\}\right)= & \Pi_{D d}^{\text {polar }}(s)+\frac{m^{D-2}}{(2 \pi)^{(D / 2)-2 s+4} \Gamma(s)} \\
& \times\left[2 W_{D d}\left(s ;\left\{b_{i}\right\}\right)-(s-1) W_{D d}\left(s-1 ;\left\{b_{i}\right\}\right)\right],
\end{aligned}
$$

where

$$
\Pi_{D d}^{\text {polar }}(s)=\frac{m^{D-2} \pi^{D / 2}(s-1-D)}{(2 \pi)^{D-2 s+4} \Gamma(s)} \Gamma\left(s-1-\frac{D}{2}\right)
$$

and the functions $W_{D d}\left(\mu ;\left\{b_{i}\right\}\right)$ are given by Eq. (13). We notice that the first term in this expression for $\Pi_{D d}\left(s ;\left\{b_{i}\right\}\right)$, $\Pi_{D d}^{\text {polar }}(s)$, does not depend on parameters $b_{i}$, that is, it is independent of the compactification lengths $L_{i}(i=$ $1, \ldots, d)$. At $s=2$, because of the poles of the $\Gamma$-function, such a term is divergent for even dimensions $D \geq 2$.

In order to obtain a finite single bubble function, we shall use a modified minimal subtraction scheme, where terms to be subtracted have poles appearing at the physical value $s=2$. Thus, the polar parcel given by Eq. (16) will be suppressed and, for the sake of uniformity, this term is also subtracted in the case of odd dimensions, where no pole of the $\Gamma$-function is present; in such a situation, we perform a finite subtraction. In this way, using the same notation, we define the finite one-loop diagram by the relation

$$
\Pi_{D d}\left(\left\{b_{i}\right\}\right)=\left.\left\{\Pi_{D d}\left(s ;\left\{b_{i}\right\}\right)-\Pi_{D d}^{\text {polar }}(s)\right\}\right|_{s=2} .
$$

Therefore, the finite one-loop diagram, which depends on the compactification lengths $L_{i}$ and arises from the regular part of the analytical extension of the Epstein-Hurwitz zeta-functions, is given by

$$
\Pi_{D d}\left(\left\{b_{i}\right\}\right)=\frac{m^{D-2}}{(2 \pi)^{D / 2}}\left[2 W_{D d}\left(2 ;\left\{b_{i}\right\}\right)-W_{D d}\left(1 ;\left\{b_{i}\right\}\right)\right] .
$$


From now on, we shall deal only with finite quantities that are obtained following this subtraction prescription. Notice that, replacing $b_{i}$ by $\left(m L_{i}\right)^{-2}$ in the above expression, we recover explicitly $\Pi_{D d}\left(\left\{L_{i}\right\}\right)$. Now, we proceed to analyze the behavior of the large- $N$ coupling constant in various cases.

\section{COUPLING CONSTANT IN THE LARGE- $N$ LIMIT}

In field theories with four-fermion interactions, the coupling constant is defined in terms of the four-point function at fixed external momenta; here we choose $\mathbf{p}=0$. In this situation, the coupling constant can be interpreted as measuring the strength of the interaction between the fermions. The large- $N$ (\{ $\left.b_{i}\right\}$-dependent $)$ coupling constant, for $d(\leq D)$ compactified dimensions, is then obtained by substituting $\Pi_{D d}\left(\left\{b_{i}\right\}\right)$ into Eq. (3) and taking the limit $N \rightarrow \infty, u \rightarrow 0$, with $N u=\lambda$ fixed, we get

$$
\begin{aligned}
g_{D d}\left(\left\{b_{i}\right\}, \lambda\right) & =\lim _{N u=\lambda}\left[N \Gamma_{D d}^{(4)}\left(0,\left\{b_{i}\right\}, u\right)\right] \\
& =\frac{\lambda}{1+\lambda \prod_{D d}\left(\left\{b_{i}\right\}\right)} .
\end{aligned}
$$

It is clear that, while $g_{D d}\left(\left\{b_{i}\right\}, \lambda\right)$ depends on the value of the fixed coupling constant $\lambda$ in a direct way, its dependence on the compactifiation lengths is dictated by the behavior of $\Pi_{D d}$ as $\left\{b_{i}\right\}$ is varied. The dependence of $g_{D d}$ on $\left\{L_{i}\right\}$ and $\lambda$ is the main point to be discussed in the subsequent analysis.

Limiting behaviors of the finite coupling constant $g_{D d}$ can be readily obtained from the fact that $\Pi_{D d}$ depends on $\left\{b_{i}\right\}$ through the Bessel functions of the third kind appearing in the definition of the functions $F_{D j}$, Eq. (14). First, if we let all the compactification lengths tend to infinity, that is $\left\{b_{i} \rightarrow 0\right\}$, thus reducing the problem to the free space at $T=0$, then $\Pi_{D d} \rightarrow 0$ and we obtain, consistently, that

$$
\lim _{\left\{L_{i} \rightarrow \infty\right\}} g_{D d}\left(\left\{b_{i}\right\}, \lambda\right)=\lambda,
$$

where $\lambda$ is the fixed coupling constant in free space at zero temperature. This is a consequence of the fact that $K_{\nu}(z \rightarrow \infty) \rightarrow 0$, for $\nu$ integer or half-integer. In the opposite limit, for any $b_{i}$ tending to $\infty$ (that is, if any compactification length $L_{i}$ goes to 0 ), the single bubble diagram $\Pi_{D d} \rightarrow \infty$, since $K_{\nu}(z) \rightarrow 0$ as $z \rightarrow \infty$. This implies that the effective coupling constant $g_{D d}$ vanishes, irrespective of the value of $\lambda$, suggesting that the system presents an asymptotic-freedom type of behavior for short distances and/or for high temperatures.

From the extreme limits considered above two situations may emerge, as one changes the compactification lengths from 0 to $\infty$ : either $\Pi_{D d}$ varies from $\infty$ to 0 through positive values, or $\Pi_{D d}$ reaches 0 before tending to 0 through negative values. The latter case, which may actually happen, would lead to an interesting situation where a divergence of the effective coupling constant would appear at finite values of the lengths $L_{i}$. This possibility, and its consequences, will be investigated explicitly in the following subsections, considering the compactified GN model at $T=0$ for space-time dimensions $D=2,3,4$. The discussion of finite-temperature effects is postponed to Sec. IV.

\section{A. Two-dimensional compactified GN model at $\boldsymbol{T}=\mathbf{0}$}

For $D=2$ and $d=1$ (two-dimensional space-time with the spatial coordinate compactified), we put $b_{1}=(m L)^{-2}$ in Eqs. (13) and (14), then Eq. (18) becomes

$$
\Pi_{21}(L)=2 E_{1}(2 m L)-E_{1}(m L),
$$

where the function $E_{1}(x)$ is defined by

$$
E_{1}(x)=\frac{1}{\pi} \sum_{n=1}^{\infty}\left[-K_{0}(x n)+(x n) K_{1}(x n)\right] .
$$

Notice that $\Pi$ is dimensionless for $D=2$.

We can calculate $\Pi_{21}(L)$ numerically by truncating the series appearing in the definition of the function $E_{1}(y)$, Eq. (22), at some value $n=M$. For moderate and large values of $m L$ (say, $m L \geqslant 0.5$ ), one can take for $M$ a relatively small value; e.g., for $m L=0.5$, choosing $M=36$ already leads to the correct value of $\Pi_{21}$ to six decimal places. As $m L$ increases, the value of $M$ can be made smaller to give the same precision. However, since the functions $K_{0}(z)$ and $K_{1}(z)$ diverge for $z \rightarrow 0$ and the summation involves positive and negative parcels, the calculation of $\Pi_{21}$ for small values of $m L$ requires large values of $M$; for $m L=0.005$, we need $M \approx 4500$ to obtain $\Pi_{21}$ to six decimal places. Fortunately, the relevant behavior of $\Pi_{21}(L)$ appears for moderate values of $m L$ so that the numerical calculations are carried out in a short computational time with very good precision.

The function $\Pi_{21}(L)$ is plotted as a function of $m L$ in Fig. 1. From this figure and the numerical treatment of Eq. (21), we infer that $\Pi_{21}(L)$ diverges $(\rightarrow+\infty)$ when $L \rightarrow 0$ and tends to 0 , through negative values, as $L \rightarrow \infty$.

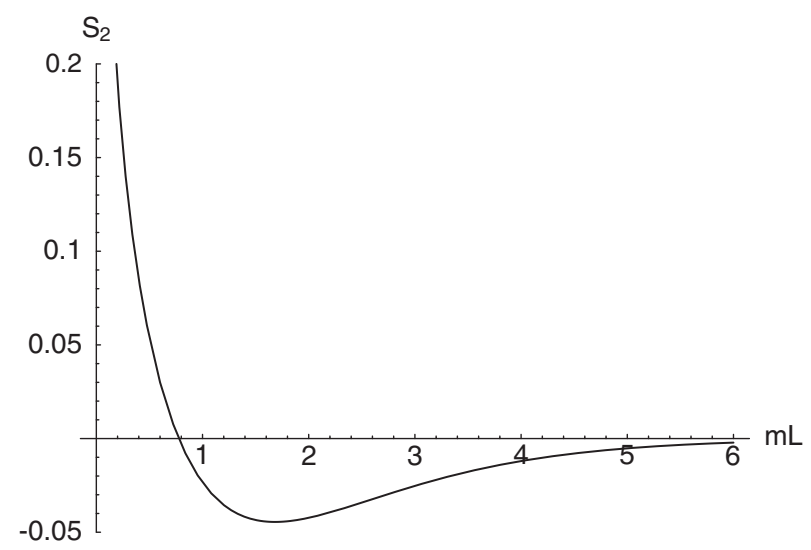

FIG. 1. Plot of $S_{2}=\Pi_{21}(L)$ as a function of $m L$. 
Also, we find that $\Pi_{21}(L)$ vanishes for a specific value of $L$, which we denote by $L_{\min }^{(2)}$, being negative for all $L>L_{\min }^{(2)}$, and assumes a minimum (negative) value at a value of $L$ denoted by $L_{\max }^{(2)}$, for reasons that will be clarified later. Numerically, it is found that $L_{\min }^{(2)} \simeq$ $0.78 \mathrm{~m}^{-1}, L_{\max }^{(2)} \simeq 1.68 \mathrm{~m}^{-1}$ and $\Pi_{21}^{\min } \simeq-0.0445$. This behavior of $\Pi_{21}$ as $L$ changes, particularly the fact that $\Pi_{21}(L)<0$ for $L>L_{\min }^{(2)}$, leads to remarkable properties of the large- $N$ coupling constant $g_{21}(L, \lambda)$. It is important to point out that such a dependence of the polarization on $L$ is a direct consequence of the use of antiperiodic boundary conditions for the spatial compactification. Taking periodic boundary conditions (PBC), one would obtain $\Pi_{21}^{\mathrm{PBC}}(L)=$ $E_{1}(m L) / 4$, which is positive for all values of $L$, and so no significant size-effect would exist.

Recall that, in the present case, Eq. (19) becomes

$$
g_{21}(L, \lambda)=\frac{\lambda}{1+\lambda \Pi_{21}(L)} .
$$

The divergence of $\Pi_{21}(L)$ as $L \rightarrow 0$ ensures that, independently of the value of $\lambda, g_{21}(L, \lambda)$ approaches 0 in this limit and, therefore, the system presents a kind of asymptoticfreedom behavior for short distances. On the other hand, since $\Pi_{21}(L)$ assumes negative values for $L>L_{\min }^{(2)}$, the denominator of Eq. (23) will vanish at a finite value of $L$ if $\lambda$ is sufficiently high. This means that, starting from a low value of $L$ (within the region of asymptotic freedom) and increasing the size of the system, $g_{21}$ will diverge at a finite value of $L, L_{c}^{(2)}(\lambda)$, if $\lambda$ is greater than the "critical value" $\lambda_{c}^{(2)}=\left(-\Pi_{21}^{\min }\right)^{-1} \simeq 22.47$. We interpret this result by stating that, in the strong-coupling regime $\left(\lambda \geq \lambda_{c}^{(2)}\right)$ the system gets spatially confined in a segment of length $L_{c}^{(2)}(\lambda)$. The behavior of the $L$-dependent coupling constant as a function of $m L$ is illustrated in Fig. 2, for some values of the fixed coupling constant $\lambda$.

It should be emphasized that we are treating the massive GN model with an arbitrary (but fixed) fermion mass. In this case, the model does not possess chiral symmetry which is explicitly broken. Since we do not expect that this symmetry appears beyond the critical value (with the

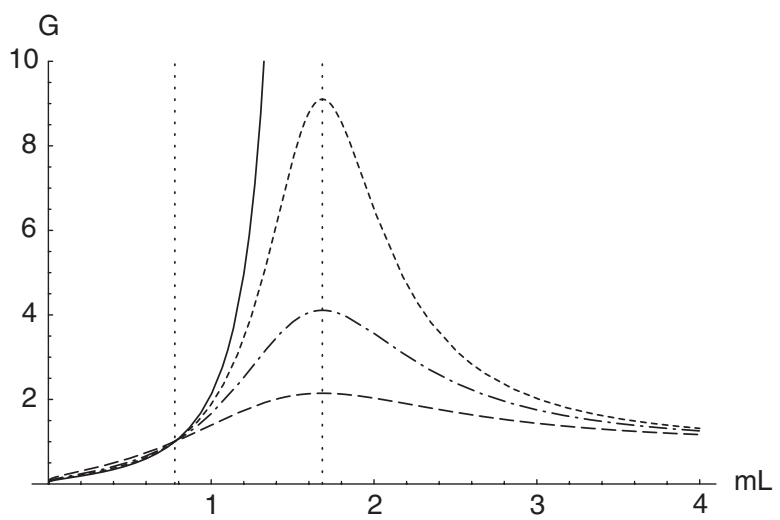

FIG. 2. Plots of the relative effective coupling constant, $G=$ $g_{21}(L, \lambda) / \lambda$, as a function of $m L$ for some values of $\lambda: 12.0$ (long-dashed line), 17.0 (dot-dashed line), 20.0 (short-dashed line), and 22.5 (solid line). The dotted vertical lines, passing by $L_{\min }^{(2)} \simeq 0.78 \mathrm{~m}^{-1}$ and $L_{\max }^{(2)} \simeq 1.68 \mathrm{~m}^{-1}$, are plotted as a visual guide.

radiatively corrected mass vanishing identically), the instability indicated by the divergence of the coupling constant is interpreted as signaling a spatial confining transition.

For $\lambda=\lambda_{c}^{(2)}$, by definition, the divergence of $g_{21}(L, \lambda)$ is reached as $L$ approaches the value that makes $\Pi_{21}$ minimal, which we have denoted by $L_{\max }^{(2)}$. In the other limit, since $g_{21}^{-1}(L, \lambda \rightarrow \infty)=\Pi_{21}(L), L_{c}^{(2)}(\lambda)$ tends to $L_{\min }^{(2)}$, the zero of $\Pi_{21}(L)$, as $\lambda \rightarrow \infty$. In other words, the confining length $L_{c}^{(2)}(\lambda)$ decreases from the maximum value $L_{\max }^{(2)}$, when $\lambda=\lambda_{c}^{(2)}$, tending to the lower bound $L_{\min }^{(2)}$ in the limit $\lambda \rightarrow \infty$. The behavior of $L_{c}^{(2)}$, as a function of $\lambda$, will be presented later.

\section{B. Compactified three-dimensional GN model at $\boldsymbol{T}=\mathbf{0}$}

For the three-dimensional model at zero temperature with two compactified dimensions $(d=2)$, denoting the compactification lengths associated with the two spatial coordinates $x_{1}$ and $x_{2}$ by $L_{1}$ and $L_{2}\left(\mathrm{~m}^{-1} / \sqrt{b_{1}}\right.$ and $m^{-1} / \sqrt{b_{2}}$, respectively), formulas in Eq. (13) and (18) give

$$
\begin{aligned}
\Pi_{32} \mu\left(b_{1}, b_{2}\right)= & \frac{m}{\sqrt{2} \pi^{3 / 2}}\left[2 F_{31}\left(2 ; b_{1}\right)-F_{31}\left(2 ; 4 b_{1}\right)+2 F_{31}\left(2 ; b_{2}\right)-F_{31}\left(2 ; 4 b_{2}\right)-2 F_{31}\left(1 ; b_{2}\right)-2 F_{31}\left(1 ; b_{1}\right)\right. \\
& +F_{31}\left(1 ; 4 b_{1}\right)+F_{31}\left(1 ; 4 b_{2}\right)+4 F_{32}\left(2 ; b_{1}, b_{2}\right)-2 F_{32}\left(2 ; 4 b_{1}, b_{2}\right)-2 F_{32}\left(2 ; b_{1}, 4 b_{2}\right)+F_{32}\left(2 ; 4 b_{1}, 4 b_{2}\right) \\
& \left.-4 F_{32}\left(1 ; b_{1}, b_{2}\right)+2 F_{32}\left(1 ; 4 b_{1}, b_{2}\right)+2 F_{32}\left(1 ; b_{1}, 4 b_{2}\right)-F_{32}\left(1 ; 4 b_{1}, 4 b_{2}\right)\right] .
\end{aligned}
$$

The functions $F_{3 j}(j=1,2)$, specified in Eq. (14), involve the Bessel functions of order $\pm \frac{1}{2}$, which are expressed in terms of elementary functions,

$$
K_{ \pm \frac{1}{2}}(z)=\sqrt{\pi} \frac{\exp (-z)}{\sqrt{2 z}} .
$$

Thus, the series defining the functions $F_{3 j}$, for both $j=$ 1,2 , are geometric series which can be summed up. Using Eq. (25) in the expression of Eq. (14) and replacing $b_{i}$ by $L_{i}^{-2}$ (which corresponds to taking all the compactification lengths measured in units of $\mathrm{m}^{-1}$, as will be done from now on), we obtain 


$$
\begin{aligned}
\Pi_{32}\left(L_{1}, L_{2}\right)= & \frac{m}{2 \pi}\left[\frac{1}{L_{1}} \log \left(1+e^{-L_{1}}\right)-\frac{1}{1+e^{L_{1}}}\right. \\
& \left.+\frac{1}{L_{2}} \log \left(1+e^{-L_{2}}\right)+\frac{1}{1+e^{L_{2}}}\right] \\
& +\frac{m}{\pi}\left[G_{2}\left(L_{1}, L_{2}\right)-2 G_{2}\left(L_{1}, 2 L_{2}\right)\right. \\
& \left.-2 G_{2}\left(2 L_{1}, L_{2}\right)+4 G_{2}\left(2 L_{1}, 2 L_{2}\right)\right],
\end{aligned}
$$

where the function $G_{2}(x, y)$ is defined by

$$
\begin{aligned}
G_{2}(x, y)= & \sum_{n, l=1}^{\infty} \exp \left(-\sqrt{x^{2} n^{2}+y^{2} l^{2}}\right) \\
& \times\left[1-\frac{1}{\sqrt{x^{2} n^{2}+y^{2} l^{2}}}\right] .
\end{aligned}
$$

The numerical computation of $\Pi_{32}\left(L_{1}, L_{2}\right)$ is greatly facilitated by the fact that the double series defining the function $G_{2}(y, z)$ is rapidly convergent. The need of truncating the summations at a larger value $n=M$ when $L_{1}$ and $L_{2}$ are very small, as in the case of $D=2$, still exists but to a much less extent.

It is to be noticed that if either compactification length $L_{1}$ or $L_{2}$ tends to $\infty$, all terms depending on it disappear from Eq. (26) and we regain the finite bubble diagram for the case where only one spatial dimension is compactified in the three-dimensional model [35]. Now, if both $L_{1}$ and $L_{2}$ tend simultaneously to $\infty, \Pi_{32}$ goes to zero and $g_{32} \rightarrow \lambda$, as expected. On the other hand, if either $L_{1}$ or $L_{2}$ tends to $0, \Pi_{32} \rightarrow+\infty$ implying that the system gets asymptotically free, with the effective coupling constant vanishing in this limit. The overall behavior of the bubble diagram is illustrated in Fig. 3, where we draw the contour plots of $\Pi_{32}\left(L_{1}, L_{2}\right) / m$. The solid line in Fig. 3 is the locus of the points such that $\Pi_{32}\left(L_{1}, L_{2}\right) / m=0$, which for large $L_{1}\left(L_{2}\right)$ approaches the straight line $L_{1}=1.14 \mathrm{~m}^{-1}\left(L_{2}=\right.$ $\left.1.14 \mathrm{~m}^{-1}\right) ; \Pi_{32}\left(L_{1}, L_{2}\right)$ is positive below this curve, negative above it, and reaches an absolute minimum, $\Pi_{32}^{\min } \simeq-0.00986 \mathrm{~m}$, at the point $L_{1}=L_{2} \simeq 2.10 \mathrm{~m}^{-1}$.

The fact that $\Pi_{32}$ assumes negative values in the whole region of the parameter space $\left(L_{1}, L_{2}\right)$ above the full line in Fig. 3 implies that, for large enough values of $\lambda, g_{32}$ will diverge at finite values of $L_{i}, i=1,2$. However, to avoid unnecessary complication, our analysis is restricted to the case where the system is confined within a square of size $L$, by considering $L_{1}=L_{2}=L$. In other words, we shall concentrate on the behavior of $\Pi_{32}$ along the diagonal of Fig. 3. We plot, in Fig. $4, S_{3}(L)=\Pi_{32}(L) / m$ as a function of $L$. We find that $S_{3}(L)$ vanishes for a specific value of $L$, $L_{\text {min }}^{(3)}$, being negative for all $L \geq L_{\min }^{(3)}$. Also, $\Pi_{32}(L)$ reaches an absolute minimum (negative) value for a value of $L$ we denote by $L_{\max }^{(3)}$. We find, numerically, that $L_{\min }^{(3)} \simeq 1.30 \mathrm{~m}^{-1}$ and $L_{\max }^{(3)} \simeq 2.10 \mathrm{~m}^{-1}$, with $\Pi_{32}^{\min } \simeq$ $-0.00986 \mathrm{~m}$, as stated before. This behavior of $\Pi_{32}(L)$

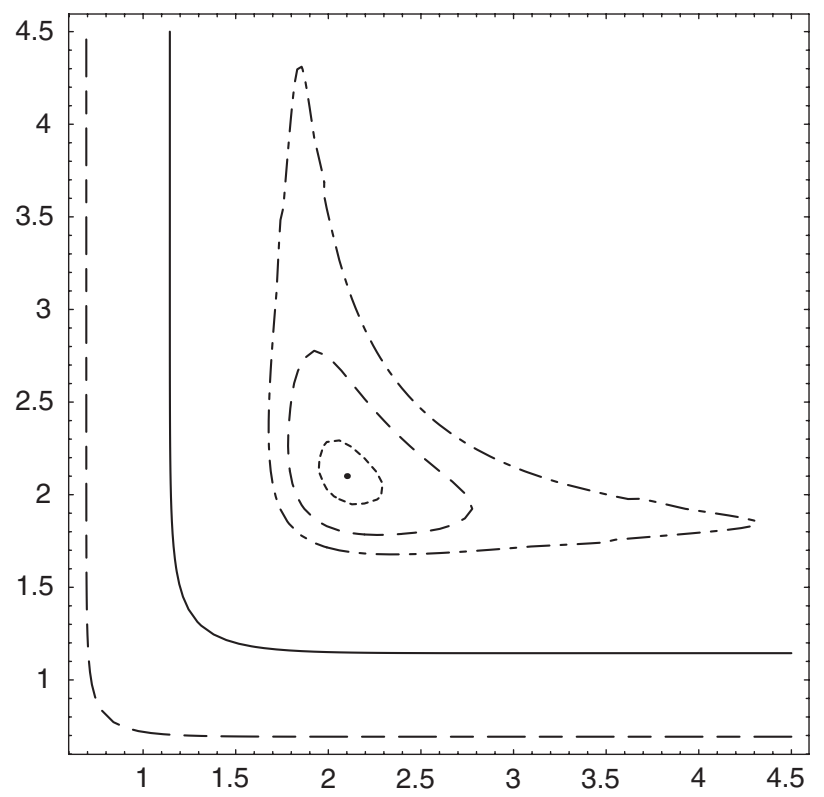

FIG. 3. Contour plots of $\Pi_{32}\left(L_{1}, L_{2}\right) / m$, with $L_{1}$ and $L_{2}$ in units of $\mathrm{m}^{-1}$. The open dashed line corresponds to $\Pi_{32}\left(L_{1}, L_{2}\right) / m=0.04$, the solid line gives the points where $\Pi_{32}=0$, while the closed curves are for negative values of $\Pi_{32} / m,-0.0091,-0.0095$ and -0.0098 (dot-dashed, dashed, and dotted lines, respectively). The dot is the location of the absolute minimum of $\Pi_{32}\left(L_{1}, L_{2}\right)$, which occurs for $L_{1}=L_{2} \simeq$ $2.1 \mathrm{~m}^{-1}$.

has profound implications on the effective coupling constant.

With $D=3$ and $d=2$, Eq. (19) is rewritten as

$$
g_{32}(L, \lambda)=\frac{\lambda}{1+\lambda \Pi_{32}(L)},
$$

and we find that, for $\lambda \geq \lambda_{c}^{(3)}=\left(-\Pi_{32}^{\min }\right)^{-1} \simeq$ $101.42 \mathrm{~m}^{-1}$, the denominator in Eq. (28) will vanish for

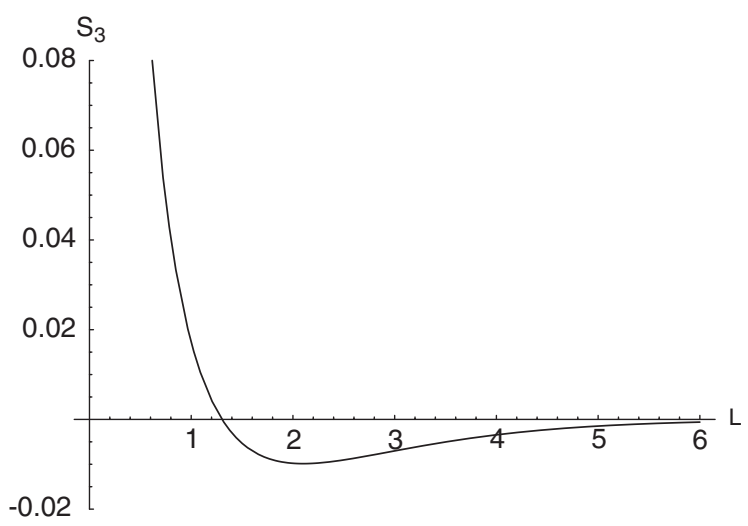

FIG. 4. Plot of $S_{3}=\Pi_{32}(L) / m$ as a function of $L$, in units of $\mathrm{m}^{-1}$. 
a finite value of $L, L_{c}^{(3)}(\lambda)$, leading to a divergence in the effective coupling constant. The behavior of the effective coupling constant as a function of $L$, for increasing values of the fixed coupling constant $\lambda$, can be illustrated showing the same pattern as that of Fig. 2 for the preceding case. Similarly, we find that the divergence occurs at $L_{c}^{(3)}(\lambda)$, which satisfies $L_{\min }^{(3)}<L_{c}^{(3)}(\lambda) \leq L_{\max }^{(3)}$. Again, we interpret such a result by considering the system spatially confined in the sense that, starting with small $L$ (in the region of asymptotic freedom), the size of the square cannot increase above $L_{c}^{(3)}(\lambda)$, since $g_{32}(L, \lambda) \rightarrow \infty$ as $L \rightarrow L_{c}^{(3)}(\lambda)$.

\section{C. $D=\mathbf{4}$ case at zero temperature}

In the case of the four-dimensional GN model with all three spatial coordinates compactified, replacing $b_{i}$ by $L_{i}^{-2}$ (again, $L_{i}$ measured in units of $\mathrm{m}^{-1}$ ) into Eqs. (13), (14), and (18), gives

$$
\begin{aligned}
\Pi_{43}\left(\left\{L_{i}\right\}\right)= & m^{2}\left[2 H_{1}\left(2 L_{1}\right)+2 H_{1}\left(2 L_{2}\right)+2 H_{1}\left(2 L_{3}\right)-H_{1}\left(L_{1}\right)-H_{1}\left(L_{2}\right)-H_{1}\left(L_{3}\right)+2 H_{2}\left(L_{1}, L_{2}\right)+2 H_{2}\left(L_{1}, L_{3}\right)\right. \\
& +2 H_{2}\left(L_{2}, L_{3}\right)-4 H_{2}\left(L_{1}, 2 L_{2}\right)-4 H_{2}\left(L_{1}, 2 L_{3}\right)-4 H_{2}\left(2 L_{1}, L_{2}\right)-4 H_{2}\left(2 L_{1}, L_{3}\right)-4 H_{2}\left(L_{2}, 2 L_{3}\right) \\
& -4 H_{2}\left(2 L_{2}, L_{3}\right)+8 H_{2}\left(2 L_{1}, 2 L_{2}\right)+8 H_{2}\left(2 L_{1}, 2 L_{3}\right)+8 H_{2}\left(2 L_{2}, 2 L_{3}\right)-4 H_{3}\left(L_{1}, L_{2}, L_{3}\right)+8 H_{3}\left(2 L_{1}, L_{2}, L_{3}\right) \\
& +8 H_{3}\left(L_{1}, 2 L_{2}, L_{3}\right)+8 H_{3}\left(L_{1}, L_{2}, 2 L_{3}\right)-16 H_{3}\left(2 L_{1}, 2 L_{2}, L_{3}\right)-16 H_{3}\left(2 L_{1}, L_{2}, 2 L_{3}\right) \\
& \left.-16 H_{3}\left(L_{1}, 2 L_{2}, 2 L_{3}\right)+32 H_{3}\left(2 L_{1}, 2 L_{2}, 2 L_{3}\right)\right]
\end{aligned}
$$

where the functions $H_{j}, j=1,2,3$, are defined by

$$
\begin{gathered}
H_{1}(x)=\frac{1}{2 \pi^{2}} \sum_{n=1}^{\infty}\left[K_{0}(x n)-\frac{K_{1}(x n)}{(x n)}\right], \\
H_{2}(x, y)=\frac{1}{2 \pi^{2}} \sum_{n, l=1}^{\infty}\left[K_{0}\left(\sqrt{x^{2} n^{2}+y^{2} l^{2}}\right)\right. \\
-\frac{K_{1}\left(\sqrt{x^{2} n^{2}+y^{2} l^{2}}\right)}{\left.\left(\sqrt{x^{2} n^{2}+y^{2} l^{2}}\right)\right],} \\
H_{3}(x, y, z)=\frac{1}{2 \pi^{2}} \sum_{n, l, r=1}^{\infty}\left[K_{0}\left(\sqrt{x^{2} n^{2}+y^{2} l^{2}+z^{2} r^{2}}\right)\right. \\
\left.-\frac{K_{1}\left(\sqrt{x^{2} n^{2}+y^{2} l^{2}}\right)}{\left(\sqrt{x^{2} n^{2}+y^{2} l^{2}+z^{2} r^{2}}\right)}\right] .
\end{gathered}
$$

Results for one or two compactified dimensions are obtained from Eq. (29) if two or one of the compactification lengths become infinite. For example, taking $L_{2}$, $L_{3} \rightarrow \infty$, we get $\Pi_{41}\left(L_{1}\right)=m^{2}\left[2 H_{1}\left(2 L_{1}\right)-H_{1}\left(L_{1}\right)\right]$, which assumes the minimum value $\Pi_{41}^{\min } \simeq-0.001866 \mathrm{~m}^{2}$ at $L=L_{\max }^{(41)} \simeq 2.01 \mathrm{~m}^{-1}$, leading to the critical value $\lambda_{c}^{(41)} \simeq$ $535.91 \mathrm{~m}^{-2}$, and vanishes at $L=L_{\min }^{(41)} \simeq 1.43 \mathrm{~m}^{-1}$. The analysis made in Ref. [35] can be extended to this case of the four-dimensional space with only one spatial coordinate compactified. Similarly, we could discuss the situation with two compactified spatial dimensions.

Here, instead of dealing with all possibilities, we concentrate on the case where the system is confined to a cubic box, that is, we take $L_{1}=L_{2}=L_{3}=L$. With equal compactification lengths, Eq. (29) becomes

$$
\begin{aligned}
\Pi_{43}(L)= & m^{2}\left[6 H_{1}(2 L)-3 H_{1}(L)+6 H_{2}(L, L)\right. \\
& -24 H_{2}(L, 2 L)+24 H_{2}(2 L, 2 L) \\
& -4 H_{3}(L, L, L)+24 H_{3}(L, L, 2 L) \\
& \left.-48 H_{3}(L, 2 L, 2 L)+32 H_{3}(2 L, 2 L, 2 L)\right] .
\end{aligned}
$$

This quantity is plotted in Fig. 5, which shows that it has the same behavior as its counterparts for $D=2$ and $D=3$. We find numerically that $\Pi_{43}(L)$ vanishes for $L=$ $L_{\min }^{(4)} \simeq 1.68 \mathrm{~m}^{-1}$, being negative for $L>L_{\min }^{(4)}$, and assumes the minimum value, $\Pi_{43}^{\min } \simeq-0.0022751 \mathrm{~m}^{2}$, when $L=L_{\max }^{(4)} \simeq 2.37 \mathrm{~m}^{-1}$.

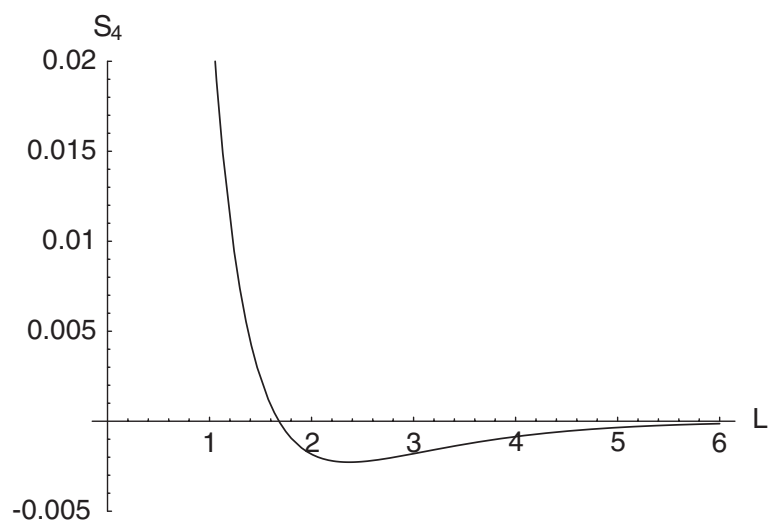

FIG. 5. Plot of $S_{4}=\Pi_{43}(L) / \mathrm{m}^{2}$ as a function of $L$, in units of $\mathrm{m}^{-1}$. 
As in the other cases, the large- $N$ coupling constant,

$$
g_{43}(L, \lambda)=\frac{\lambda}{1+\lambda \Pi_{43}(L)},
$$

diverges at a finite value of $L, L_{c}^{(4)}(\lambda)$, if $\lambda \geq \lambda_{c}^{(4)}=$ $-\left(\Pi_{43}^{\min }\right)^{-1} \simeq 439.54 \mathrm{~m}^{-2}$, meaning that the system gets confined in a cubic box of edge $L_{c}^{(4)}(\lambda)$ which is bounded in the interval between the values $L_{\min }^{(4)}$ and $L_{\max }^{(4)}$.

\section{Dependence of $L_{c}^{(D)}$ on $\lambda$}

For the cases we have analyzed above, namely $D=$ 2,3,4 with all spatial coordinates compactified and $L_{1}=\cdots=L_{D-1}=L$, we find that the confining length $L_{c}^{(D)}(\lambda)$ lies in a finite interval,

$$
L_{c}^{(D)}(\lambda) \in\left(L_{\min }^{(D)}, L_{\max }^{(D)}\right],
$$

where the maximum value corresponds to $\lambda_{c}^{(D)}$, the minimum value of the fixed coupling constant $\lambda$ allowing spatial confinement, while $L_{\min }^{(D)}$ sets the bound as $\lambda \rightarrow \infty$. Then, a question emerges of how $L_{c}^{(D)}(\lambda)$ changes as $\lambda$ increases from $\lambda_{c}^{(D)}$ to infinity.

For a given value of $\lambda\left(\geq \lambda_{c}^{(D)}\right)$, the confining length $L_{c}^{(D)}(\lambda)$ can be found numerically by determining the smallest root of the equation

$$
g_{D D-1}^{-1}(L, \lambda)=\frac{1}{\lambda}\left[1+\lambda \Pi_{D D-1}(L)\right]=0 .
$$

That is, following the interpretation provided before, starting from small values of $L$, the first value at which $g_{D D-1}^{-1}$ vanishes does provide the confining length of the system, $L_{c}^{(D)}(\lambda)$. In Fig. 6, we plot $L_{c}^{(D)}(\lambda)$ as a function of $l=\lambda / \lambda_{c}^{(D)}$, for the cases $D=2,3,4$.

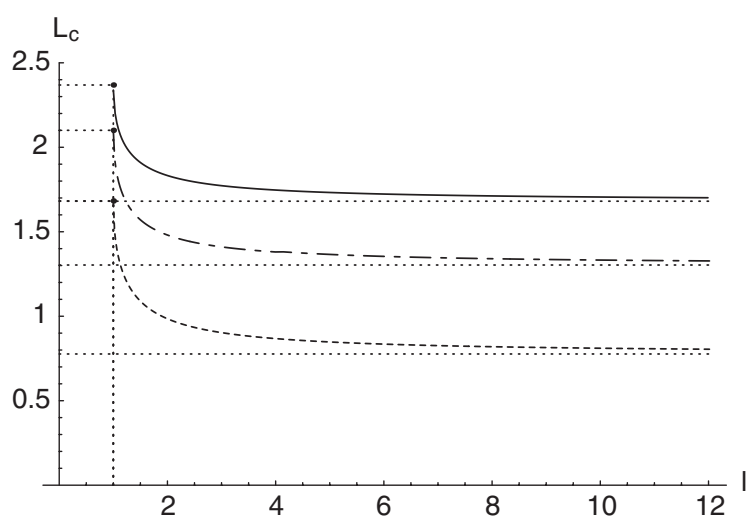

FIG. 6. Plot of the confining length (in units of $\mathrm{m}^{-1}$ ), as a function of $l=\lambda / \lambda_{c}^{(D)}$, for $D=2,3,4$ (dashed, dot-dashed, and solid lines, respectively); the horizontal dotted lines correspond to the limiting values $L_{\min }^{(D)}$ and $L_{\max }^{(D)}$ (given in the text), plotted as a visual guide.

\section{COMPACTIFIED GN MODEL AT FINITE TEMPERATURE}

We shall now consider the effect of raising the temperature on the effective coupling constant for the GN model with all spatial dimensions compactified. Finitetemperature effects are introduced through the compactification of the time coordinate, with the compactification "length" given by $L_{D}=\beta=1 / T$, where $T$ is the temperature. It is important to emphasize that, although in a Euclidean theory, time and space coordinates are treated on the same footing, the interpretations of their compactifications are rather distinct; while compactification of spatial dimensions can be thought as describing confined fields, time compactification corresponds to taking the system in thermal equilibrium at temperature $\beta^{-1}$.

We generally expect that the dependence of $\Pi_{D D}$ and $g_{D D}$ on $\beta$ should follow similar patterns as that for the dependence on $L$. As in the case where any compactification length $L_{i}$ tends to zero, we find that $\Pi_{D D}(L, \beta) \rightarrow \infty$ as $\beta \rightarrow 0(T \rightarrow \infty)$, implying that $g_{D D} \rightarrow 0$ independently of the value of the fixed coupling constant $\lambda$. This means that we have an asymptotic-freedom behavior for very high temperatures.

For $\beta \rightarrow \infty(T \rightarrow 0)$, the behavior of $\Pi_{D D-1}(L)$ has been described earlier: for sufficiently high values of $\lambda$, the system is confined in a $(D-1)$-dimensional cube of edge $L_{c}^{(D)}$. Based on these observations, we expect that, starting from the compactified model at $T=0$ with $\lambda \geq \lambda_{c}^{(D)}$, raising the temperature will lead to the suppression of the divergence of $g_{D D}$ and the consequent spatial deconfinement of the system, at a specific value of the temperature, $T_{d}^{(D)}$. The way such a "deconfining" transition occurs and the determination of the deconfining temperature for the cases of $D=2,3,4$ are the points addressed in the next subsections.

\section{A. Two-dimensional compactified GN model at $\boldsymbol{T} \neq \mathbf{0}$}

To account for the effect of finite temperature on the two-dimensional compactified GN model [59], we take the second Euclidean coordinate (the imaginary time, $x_{2}$ ) compactified in a length $L_{2}=\beta=1 / T$. In this case, replacing $b_{1}=L^{-2}$ and $b_{2}=\beta^{-2}(L$ and $\beta$ measured in units of $\mathrm{m}^{-1}$ ) into Eqs. (13), (14), and (18), the $L$ and $\beta$-dependent bubble diagram can be written as

$$
\begin{aligned}
\Pi_{22}(L, \beta)= & 2 E_{1}(2 L)-E_{1}(L)+2 E_{1}(2 \beta)-E_{1}(\beta) \\
& +2 E_{2}(L, \beta)-4 E_{2}(2 L, \beta)-4 E_{2}(L, 2 \beta) \\
& +8 E_{2}(2 L, 2 \beta),
\end{aligned}
$$

where the function $E_{1}(x)$ is given by Eq. (22) and the function $E_{2}(x, y)$ is defined by 


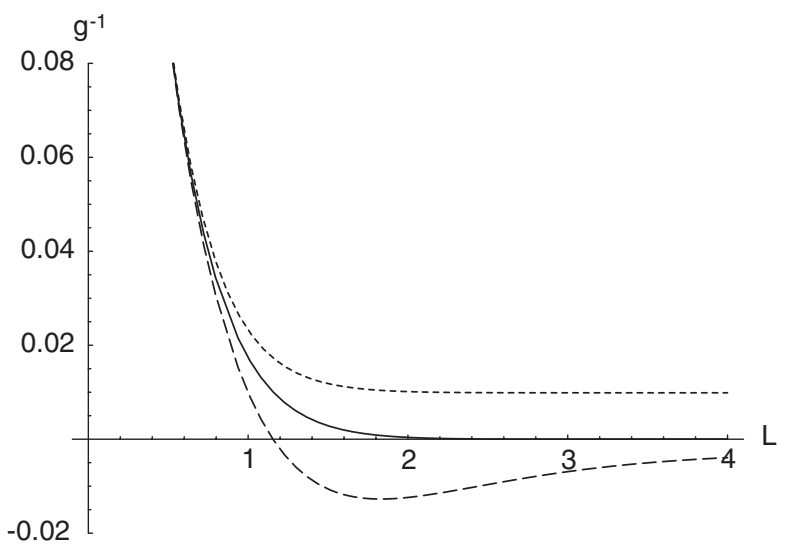

FIG. 7. Inverse of the effective coupling constant $g_{22}^{-1}$, with $\lambda=30$ fixed, as a function of $L$ (in units of $\mathrm{m}^{-1}$ ), for some values of $\beta$ (in units of $\mathrm{m}^{-1}$ ): 2.4, 1.15 , and 1.0 (dashed, solid, and dotted lines, respectively).

$$
\begin{aligned}
E_{2}(x, y)= & \frac{1}{\pi} \sum_{n, l=1}^{\infty}\left[-K_{0}\left(\sqrt{x^{2} n^{2}+y^{2} l^{2}}\right)\right. \\
& \left.+\left(\sqrt{x^{2} n^{2}+y^{2} l^{2}}\right) K_{1}\left(\sqrt{x^{2} n^{2}+y^{2} l^{2}}\right)\right]
\end{aligned}
$$

We first notice that, due to the behavior of the Bessel functions $K_{0}(z)$ and $K_{1}(z)$, all $\beta$-dependent terms in Eq. (37) vanish in the limit $\beta \rightarrow \infty$ and so $\Pi_{22}(L, \beta)$ reduces to the expression for zero temperature, $\Pi_{21}(L)$. On the other hand, if $\beta \rightarrow 0, \Pi_{22}(L, \beta) \rightarrow \infty$ and, independently of the value of $\lambda$, the system becomes asymptotically free. Therefore, we expect that, within the strong-coupling regime, raising the temperature leads to the suppression of the divergence of $g_{22}$ and the disappearance of the spatial confinement. In other words, for a given value of $\lambda \geq \lambda_{c}^{(2)}$, there exists a temperature, $T_{d}^{(2)}(\lambda)$, above which $g_{22}$ has no divergence and the system is spatially deconfined.

The deconfining temperature $T_{d}^{(2)}(\lambda)$ is determined by analyzing the behavior of $g_{22}^{-1}(L, \beta, \lambda)$ as $T$ is increased. This process is illustrated in Fig. 7, where we plot $g_{22}^{-1}(L, \beta, \lambda)$ as a function of $L$, for some values of $\beta$ and a fixed value of $\lambda>\lambda_{c}^{(2)}$. For this example with $\lambda=30$, we find that the minimum value of $g_{22}^{-1}$ vanishes for $\beta=$ $\beta_{d}^{(2)} \simeq 1.15 \mathrm{~m}^{-1}$ and is positive for $\beta>\beta_{d}^{(2)}$. Thus, the deconfining temperature, for $\lambda=30$, is given by $T_{d}^{(2)}=$ $\left(\beta_{d}^{(2)}\right)^{-1} \simeq 0.87 \mathrm{~m}$. The full dependence of deconfining temperature on $\lambda$ will be discussed later.

\section{B. Three-dimensional compactified GN model at finite $T$}

We now take the time coordinate $\left(x_{3}\right)$ compactified in a length $\beta=1 / T$ to investigate the temperature effect in the three-dimensional compactified GN model. In Ref. [36] the three-dimensional model, with only one spatial dimension compactified, was treated at finite temperature [60]. Here, we deal with the fully compactified model. Taking $b_{1}=$ $b_{2}=L^{-2}$ and fixing $b_{3}=\beta^{-2}$ in Eqs. (13), (14), and (18), the $L-\beta$ dependent bubble diagram is given by

$$
\begin{aligned}
\Pi_{33}(L, \beta)= & \frac{m}{2 \pi}\left[\frac{2}{L} \log \left(1+e^{-L}\right)-\frac{2}{1+e^{L}}+\frac{1}{\beta} \log \left(1+e^{-\beta}\right)-\frac{1}{1+e^{\beta}}\right]+\frac{m}{\pi}\left[G_{2}(L, L)+2 G_{2}(L, \beta)-4 G_{2}(L, 2 L)\right. \\
& -4 G_{2}(2 L, \beta)-4 G_{2}(L, 2 \beta)+4 G_{2}(2 L, 2 L)+8 G_{2}(2 L, 2 \beta)-2 G_{3}(L, L, \beta)+4 G_{3}(L, L, 2 \beta) \\
& \left.+8 G_{3}(2 L, L, \beta)-8 G_{3}(2 L, 2 L, \beta)-16 G_{3}(2 L, L, 2 \beta)+16 G_{3}(2 L, 2 L, 2 \beta)\right]
\end{aligned}
$$

where $G_{2}(x, y)$ is given by Eq. (27) and the function $G_{3}(x, y, z)$ is defined by

$$
G_{3}(x, y, z)=\sum_{n, l, r=1}^{\infty} \exp \left(-\sqrt{x^{2} n^{2}+y^{2} l^{2}+z^{2} r^{2}}\right)\left[1-\frac{1}{\sqrt{x^{2} n^{2}+y^{2} l^{2}+z^{2} r^{2}}}\right]
$$

Notice that, taking $\beta \rightarrow \infty$, Eq. (39) reduces to $\Pi_{32}(L)$, obtained from Eq. (26) with $L_{1}=L_{2}=L$.

As before, the increase of the temperature destroys the spatial confinement that exists for $\lambda \geq \lambda_{c}^{(3)}$ at $T=0$. We can determine the deconfining temperature by searching for the value of $\beta(\lambda)$ for which the minimum of the inverse of the effective coupling constant, $g_{33}^{-1}(L, \beta, \lambda)=$ $\left(1+\lambda \Pi_{33}(L, \beta)\right) / \lambda$, vanishes. For example, taking the specific case of $\lambda=110 \mathrm{~m}^{-1}$, we find $\beta_{d}^{(3)} \simeq 1.65 \mathrm{~m}^{-1}$ which corresponds to the deconfining temperature of $T_{d}^{(3)} \simeq$ $0.61 \mathrm{~m}$; this result can be illustrated in a figure with the same pattern as that appearing in Fig. 7 for the $D=2$ case.

\section{C. $D=4$ case at $T \neq 0$}

For the fully compactified four-dimensional GN model, fixing $b_{1}=b_{2}=b_{3}=L^{-2}$ and $b_{4}=\beta^{-2}$ ( $L$ and $\beta$ in units of $\mathrm{m}^{-1}$ ), we find from Eqs. (13), (14), and (18), that 


$$
\begin{aligned}
\Pi_{44}(L, \beta)= & m^{2}\left[6 H_{1}(2 L)-3 H_{1}(L)+2 H_{1}(2 \beta)-H_{1}(\beta)+6 H_{2}(L, L)+6 H_{2}(L, \beta)-24 H_{2}(L, 2 L)-12 H_{2}(L, 2 \beta)\right. \\
& -12 H_{2}(2 L, \beta)+24 H_{2}(2 L, 2 L)+24 H_{2}(2 L, 2 \beta)-4 H_{3}(L, L, L)-12 H_{3}(L, L, \beta)+24 H_{3}(L, L, 2 L) \\
& +48 H_{3}(L, 2 L, \beta)+24 H_{3}(L, L, 2 \beta)-48 H_{3}(L, 2 L, 2 L)-48 H_{3}(2 L, 2 L, \beta)-96 H_{3}(L, 2 L, 2 \beta) \\
& +32 H_{3}(2 L, 2 L, 2 L)+96 H_{3}(2 L, 2 L, 2 \beta)+8 H_{4}(L, L, L, \beta)-48 H_{4}(L, L, 2 L, \beta)+192 H_{4}(L, 2 L, 2 L, \beta) \\
& -16 H_{4}(L, L, L, 2 \beta)+96 H_{4}(L, L, 2 L, 2 \beta)-64 H_{4}(2 L, 2 L, 2 L, \beta)-192 H_{4}(L, 2 L, 2 L, 2 \beta) \\
& \left.+128 H_{4}(2 L, 2 L, 2 L, 2 \beta)\right],
\end{aligned}
$$

where the functions $H_{1}, H_{2}$, and $H_{3}$ are given by Eqs. (30)-(32), and $H_{4}(x, y, z, w)$ is defined by

$$
\begin{aligned}
H_{4}(x, y, z, w)= & \frac{1}{2 \pi^{2}} \sum_{n, l, r, s=1}^{\infty}\left[K_{0}\left(\sqrt{x^{2} n^{2}+y^{2} l^{2}+z^{2} r^{2}+w^{2} s^{2}}\right)\right. \\
& \left.-\frac{K_{1}\left(\sqrt{x^{2} n^{2}+y^{2} l^{2}+z^{2} r^{2}+w^{2} s^{2}}\right)}{\sqrt{x^{2} n^{2}+y^{2} l^{2}+z^{2} r^{2}+w^{2} s^{2}}}\right] .
\end{aligned}
$$

Proceeding as before, we determine the deconfining temperature by searching for the value of $\beta(\lambda)$ for which the minimum of the inverse of the effective coupling constant, $g_{44}^{-1}(L, \beta, \lambda)=\left(1+\lambda \Pi_{44}(L, \beta)\right) / \lambda$, vanishes. For example, taking the specific case of $\lambda=620 \mathrm{~m}^{-2}$, we find $\beta_{d}^{(4)} \simeq 1.707 \mathrm{~m}^{-1}$ which corresponds to the deconfining temperature of $T_{d}^{(4)} \simeq 0.59 \mathrm{~m}$.

\section{Dependence of $T_{d}^{(D)}$ on $\lambda$}

We now determine the dependence of the deconfining temperature on the fixed coupling constant. For the system with all spatial dimensions compactified and at finite temperature, we have

$$
g_{D D}^{-1}(L, \beta ; \lambda)=\frac{1}{\lambda}\left[1+\lambda \Pi_{D D}(L, \beta)\right] .
$$

As discussed before, the system is deconfined at a given temperature if the minimal value of $g_{D D}^{-1}(L, \beta ; \lambda)$ with respect to changes in $L$ is positive. It was also argued that, no matter how high the value of $\lambda$ is, the system becomes deconfined above a given temperature, $T_{d}^{(D)}(\lambda)$. In fact, one expects that

$$
T_{d}^{(D)}(\lambda) \in\left[T_{\min }^{(D)}, T_{\max }^{(D)}\right),
$$

with the limiting values corresponding to $\lambda_{c}^{(D)}$ and $\lambda \rightarrow \infty$, respectively.

From Eq. (43), we find that $\min _{\{L\}} g_{D D}^{-1}(L, \beta ; \lambda)=[1+$ $\left.\lambda M_{D}(\beta)\right] / \lambda$, where we have defined the function

$$
M_{D}(\beta)=\min _{\{L\}} \Pi_{D D}(L, \beta) .
$$

For a fixed value of $\lambda$, the behavior of the minimum value of $g_{D D}^{-1}(L, \beta ; \lambda)$ relative to changes in $L$ is dictated by the function $M_{D}(\beta)$. Notice that $M_{D}(\beta \rightarrow \infty)=\Pi_{D D-1}^{R \min }=$ $-\left[\lambda_{c}^{(D)}\right]^{-1}$, while $M_{D}(\beta) \rightarrow \infty$ as $\beta \rightarrow 0$.

Consider, initially, the case $D=2$. The function $M_{2}(\beta)$ is illustrated in Fig. 8. We find that, as $\beta$ decreases from $\infty$ (i.e., $T$ increases from 0 ), the minimum value of $\Pi_{22}(L, \beta)$ (with respect to changes in $L$ ) starts to decrease from negative values, passes through the lowest value, and then starts to increase, reaching zero at a certain value of $\beta$ below which the minima of $\Pi_{22}$ are positive. Thus, for $\lambda=\lambda_{c}^{(2)}$, increasing the temperature from zero, the system remains confined until the temperature reaches the value $T_{\min }^{(2)}=\left[\beta_{\max }^{(2)}\right]^{-1} \simeq 0.65 \mathrm{~m}$, corresponding to the finite solution of the equation $1+\lambda_{c}^{(2)} M_{2}(\beta)=0, \beta=\beta_{\max }^{(2)} \simeq$ $1.54 \mathrm{~m}^{-1}$, which is indicated by the vertical dotted line in Fig. 8. Now, if we take $\lambda>\lambda_{c}^{(2)}$, the deconfining temperature is obtained from the solution of the equation $1+$ $\lambda M_{2}(\beta)=0$, which can be determined from the intercept of the horizontal line at $-\lambda^{-1}$ (lying above the line $-\left[\lambda_{c}^{(2)}\right]^{-1}$ and below the $\beta$ axis) and the graph of $M_{2}(\beta)$. Naturally, as one takes $\lambda \rightarrow \infty$, the existence of a solution of this equation requires $M_{2}(\beta) \rightarrow 0$; this lower (open) limit occurs at the value $\beta=\beta_{\min }^{(2)} \simeq 0.776 \mathrm{~m}^{-1}$, corresponding to the temperature $T_{\max }^{(2)} \simeq 1.29 \mathrm{~m}$. The dependence of the deconfining temperature $T_{d}^{(2)}$ on $\lambda$ is determined numerically. We find that, for $\lambda$ not close to $\lambda_{c}^{(2)}$ (that is, for $\left.\lambda \gtrsim 1.5 \lambda_{c}^{(2)}\right), \beta_{d}^{(2)}(\lambda)=L_{c}^{(2)}(\lambda)$ within six decimal places; this approximate equality, valid for large values of $\lambda$, is a consequence of the symmetry of the

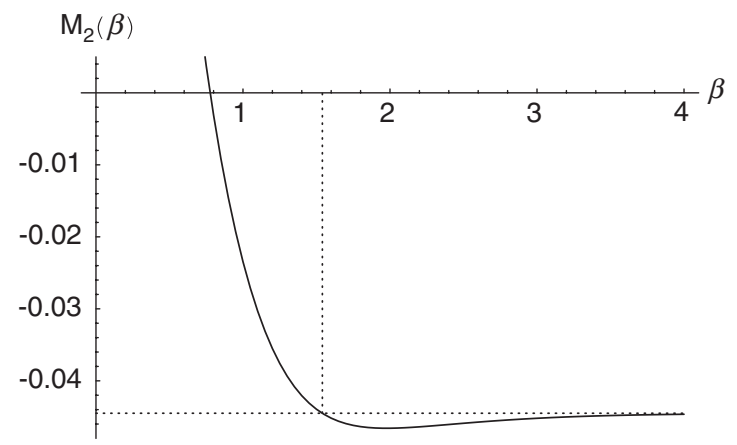

FIG. 8. Minimal values of $\Pi_{22}(L ; \beta)$ with respect of changes in $L$, as a function of $\beta$ (in units of $\mathrm{m}^{-1}$ ). The dotted horizontal line corresponds to the value $-\left[\lambda_{c}^{(2)}\right]^{-1}$. 
expression for $\Pi_{22}(L, \beta)$ by the change $L \leftrightarrow \beta$. The whole behavior of $T_{d}^{(2)}(\lambda)$ is shown in Fig. 10, together with the other cases.

It should be remarked that the absolute minimum of $\Pi_{22}(L, \beta), \quad \Pi_{22}^{\min } \simeq-0.0466$, is slightly smaller than $\Pi_{21}^{\min }$ and occurs at the point with $L=\beta \simeq 1.98 \mathrm{~m}^{-1}$. This means, as shown in Fig. 8, that the minimum value of $M_{2}(\beta)$ does not occur at zero temperature. This leads to an anomalous situation, for a small range of values of the coupling constant $\lambda\left(21.46<\lambda<\lambda_{c}^{(2)} \simeq 22.47\right)$, in which no singularity exists at $T=0$ but the equation $1+$ $\lambda M_{2}(\beta)=0$ possesses two solutions for finite values of $\beta$; this would imply that the system being unconfined at $T=0$ would get confined at a finite temperature and then become unconfined again at a smaller value of $\beta$. Such a situation, which emerges from the mathematical structure of the zeta-function regularization, has no physical meaning and will be discarded; we shall only consider the strong-coupling regime which is free from pathologies.

For $D=3$, the graph of the function $M_{3}(\beta) / m$ has a form similar to that of $M_{2}(\beta)$ (illustrated in Fig. 8) so that the same reasoning leads to the deconfining temperature $T_{d}^{(3)}(\lambda)=\left[\beta_{d}^{(3)}(\lambda)\right]^{-1}$, where $\beta_{d}^{(3)}(\lambda)$ is the finite root of the equation $1+\lambda M_{3}(\beta)=0$. In this case, the limiting values are $\beta_{\max }^{(3)} \simeq 1.85 \mathrm{~m}^{-1}$ and $\beta_{\min }^{(3)} \simeq 1.14 \mathrm{~m}^{-1}$, associated with $\lambda=\lambda_{c}^{(3)}$ and $\lambda \rightarrow \infty$, corresponding to $T_{\min }^{(3)} \simeq$ $0.54 \mathrm{~m}$ and $T_{\max }^{(3)} \simeq 0.88 \mathrm{~m}$. The plot of $T_{d}^{(3)}(\lambda)$, as a function of $l=\lambda / \lambda_{c}^{(3)}$, is also shown in Fig. 10. Distinctly from the $D=2$ case, where all the expressions are symmetric by the change $L \leftrightarrow \beta$, here we find that $\beta_{d}^{(3)}(\lambda)<L_{c}^{(3)}(\lambda)$ for all $\lambda \geq \lambda_{c}^{(3)}$, the difference being of the order of $10 \%$. The maximum value of $T_{d}^{(3)}(\simeq 0.87 \mathrm{~m})$, which occurs as $\lambda \rightarrow \infty$, corresponds to $\beta_{d}^{(3)}$ reaching the value of the minimal confining length $\left(\simeq 1.14 \mathrm{~m}^{-1}\right)$ when only one coordinate is compactified.

The $D=4$ case is more subtle due to the behavior of the function $M_{4}(\beta)$, which is presented in Fig. 9. We find that the minimum value of $\Pi_{44}(L, \beta)$ (with respect to variations of $L$ ) starts to increase from negative values as $\beta$ is diminished from $\infty$, reaches a local maximum value $\left(M_{\max } \simeq-0.0018356 \mathrm{~m}^{2}\right)$, decreases to a local minimum $\left(M_{\min } \simeq-0.002091 \mathrm{~m}^{2}\right)$ before increasing to reach zero and become positive. Therefore, for $\lambda=\lambda_{c}^{(4)}=$ $-\left[M_{4}(\beta \rightarrow \infty)\right]^{-1} \simeq 439.54 \mathrm{~m}^{-2}$, no finite solution of the equation $1+\lambda_{c}^{(4)} M_{4}(\beta)=0$ exists, which means that the system is deconfined if the temperature is greater than zero, no matter how small it is; that is, for $\lambda=\lambda_{c}^{(4)}$, spatial confinement is only possible strictly at $T=0$. For $\lambda_{c}^{(4)}<$ $\lambda<478.2 \mathrm{~m}^{-2}\left(\simeq-M_{\min }^{-1}\right)$, the equation $1+\lambda M_{4}(\beta)=$ 0 possesses one solution occurring at finite $\beta$, which could eventually be interpreted as leading to a deconfining temperature. However, if $478.2 \mathrm{~m}^{-2}<\lambda<544.8 \mathrm{~m}^{-2}$ $\left(\simeq-M_{\max }^{-1}\right)$, the equation $1+\lambda M_{4}(\beta)=0$ has three

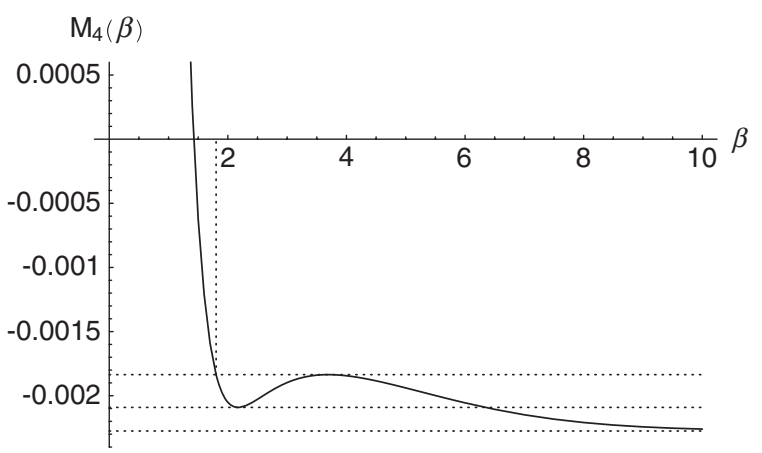

FIG. 9. Minimal values of $\Pi_{44}(L ; \beta) / m^{2}$ with respect of changes in $L$, as a function of $\beta$ (in units of $\mathrm{m}^{-1}$ ). The dotted horizontal lines correspond to the values $-\left[\lambda_{c}^{(4)}\right]^{-1}<$ $M_{\min }<M_{\max }$

distinct finite solutions. If we interpret the highest one as giving the deconfining temperature, we would have to face the puzzling situation in which the system would reenter a spatially confined phase for $\beta$ ranging between the other two smaller solutions. Such an anomalous behavior can be avoided if we redefine the strong-coupling regime by considering the range $-M_{\max }^{-1}<\lambda<\infty$.

With such a redefinition of the strong-coupling regime for $D=4$, which amounts to considering the lowest value of $\lambda$ leading to spatial confinement as being $\lambda_{c}^{(4)}=$ $-M_{\max }^{-1} \simeq 544.8 \mathrm{~m}^{-2}$, we find a well-defined deconfining temperature obtained from the intercept of the horizontal line at $-\lambda^{-1}$ and the curve $M_{4}(\beta)$. We get $\beta_{\max }^{(4)} \simeq$ $1.80 \mathrm{~m}^{-1}$ (indicated by the vertical dotted line in Fig. 9) and $\beta_{\text {min }}^{(4)} \simeq 1.43 \mathrm{~m}^{-1}$ (where $M_{4}$ vanishes), corresponding to the limits of the deconfining temperature $T_{\min }^{(4)} \simeq 0.55 \mathrm{~m}$ and $T_{\max }^{(4)} \simeq 0.70 \mathrm{~m}$, respectively. Similar to the $D=3$ case, the value of $\beta_{\min }^{(4)}$, giving the upper bound for $T_{d}^{(4)}$ $(\lambda \rightarrow \infty)$, is identical to the smallest confining length when only one spatial dimension is compactified. The overall

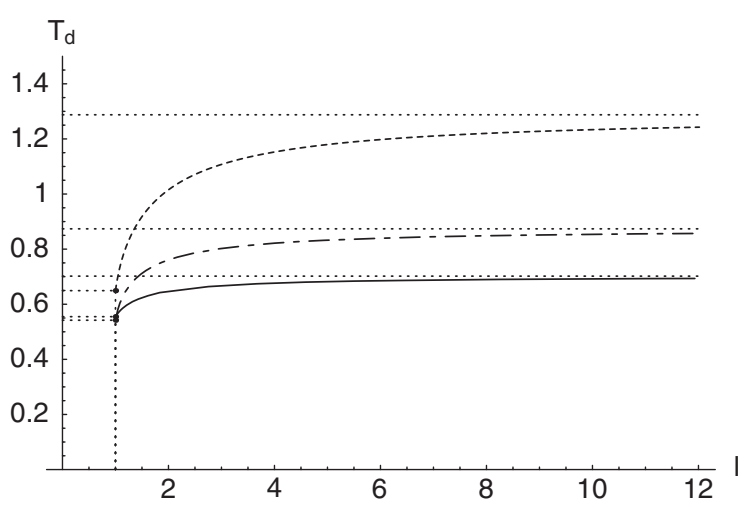

FIG. 10. Deconfining temperature $T_{d}^{(D)}(\lambda)$ (in units of m), as a function of $l=\lambda / \lambda_{c}^{(D)}$, for $D=2,3,4$ (dashed, dot-dashed, and solid lines, respectively); the horizontal dotted lines correspond to the limiting values $T_{\min }^{(D)}$ and $T_{\max }^{(D)}$ (given in the text), plotted as a visual guide. 
behavior of $T_{d}^{(4)}(\lambda)$, found numerically, is presented in Fig. 10 together with the cases of $D=2$ and 3 .

\section{CONCLUDING REMARKS}

We have used methods of quantum field theory in toroidal topologies to investigate the behavior of the $D$-dimensional massive, $N$-component, Gross-Neveu model with compactified spatial dimensions. The model is treated both at zero and finite temperatures. We calculate the large- $N$ coupling constant, $g_{D}$, as a function of the compactification length $L$, the temperature $T=\beta^{-1}$, and the fixed coupling constant $\lambda$, i.e. $g_{D}=g_{D}(L, \beta ; \lambda)$. We find that, for either $L \rightarrow 0$ or $T \rightarrow \infty$, irrespective of the value of $\lambda, g_{D}$ tends to 0 indicating that the system presents a sort of asymptotic-freedom behavior in these limits, where the effective interaction between the fermions vanishes.

For $T=0$, in the strong-coupling regime $\left(\lambda \geq \lambda_{c}^{(D)}\right)$, increasing $L$ from low values (within the asymptoticfreedom region) leads to a divergence of $g_{D}$ at a finite critical value $L=L_{c}^{(D)}(\lambda)$, signaling the existence of a second-order phase transition, as suggested by the linear response theory. Also, since we consider a four-fermion interaction model at zero chemical potential, we should not expect a first-order phase transition for any value of the parameters characterizing the system, as happens for massless models. We interpret this singularity as indicating that the system gets spatially confined in a $(D-1)$ dimensional box of edge $L_{c}^{(D)}(\lambda)$. We have shown that $L_{c}^{(D)}(\lambda)=f_{D}(\lambda) \mathrm{m}^{-1}$, where the functions $f_{D}(\lambda)$ are plotted in Fig. 6, for $D=2,3,4$.

As $T$ is raised from 0 , with $\lambda \geq \lambda_{c}^{(D)}$, the minimum value of $g_{D}^{-1}(L, \beta ; \lambda)$, with respect to changes in $L$ for fixed values of $\beta$, increases from negative values reaching 0 at a temperature $T_{d}^{(D)}(\lambda)$ above which $g_{D}$ does not present any divergence. We interpret this fact as the system being deconfined for temperatures higher than $T_{d}^{(D)}(\lambda)$. To avoid any anomalous behavior, we have redefined the value of $\lambda_{c}^{(4)}$ augmenting the lower bound which defines the strongcoupling regime when $D=4$. In any case, we find that $T_{d}^{(D)}(\lambda)=h_{D}(\lambda) \mathrm{m}$, where $h_{D}(\lambda)$ are the functions plotted in Fig. 10, for $D=2,3,4$. It is to be noted that, for the redefined value $\lambda_{c}^{(4)} \simeq 544.8 \mathrm{~m}^{-2}$, the zero-temperature maximum confining length is given by $L_{\max }^{(4)} \simeq 2.00 \mathrm{~m}^{-1}$.

It is worth emphasizing that the dependencies of $L_{c}^{(D)}$ and $T_{d}^{(D)}$ on the parameters $\lambda$ and $m$ are intrinsic results of the model; that is, they do not emerge from any adjustment. The dependence on $m$ is precisely what one expects from dimensional arguments, with $L$ and $\beta$ being proportional to $\mathrm{m}^{-1}$ in natural units. But since the functions $f_{D}(\lambda)$ and $h_{D}(\lambda)$ take on values in finite intervals (in all cases, contained in $[0.5,2.5])$, we find that extremely light fermions $(m \rightarrow 0)$ are not confined at all, while extremely heavy
TABLE I. Limiting values of $L_{c}^{(D)}(\lambda)[\mathrm{fm}]$ and $T_{d}^{(D)}(\lambda)[\mathrm{MeV}]$, for $\mathrm{m} \approx 350 \mathrm{MeV} \simeq 1.75 \mathrm{fm}^{-1}$.

\begin{tabular}{lllll}
\hline \hline$D$ & $L_{\min }^{(D)}$ & $L_{\max }^{(D)}$ & $T_{\min }^{(D)}$ & $T_{\max }^{(D)}$ \\
\hline 2 & 0.45 & 0.96 & 227 & 451 \\
3 & 0.74 & 1.2 & 189 & 304 \\
4 & 0.96 & $1.14^{\mathrm{a}}$ & $193^{\mathrm{a}}$ & 245 \\
\hline \hline
\end{tabular}

a These values of $L_{\max }^{(4)}$ and $T_{\min }^{(4)}$ correspond to the redefined value of $\lambda_{c}^{(4)}$.

ones $(m \rightarrow \infty)$ would be strictly confined in a dot, no matter what the value of $\lambda$. Also, it should be noticed that the product $\mathcal{P}_{D}(\lambda)=L_{c}^{(D)}(\lambda) T_{d}^{(D)}(\lambda)=f_{D}(\lambda) h_{D}(\lambda)$ is dimensionless and very close to the unit in the whole strong-coupling regime, $\lambda \geq \lambda_{c}^{(D)}$, irrespective of the value of $D$; actually, one finds that $\mathcal{P}_{D}(\lambda) \in(1.00,1.18)$ for all cases. Such an inverse relation between the confining length and the deconfining temperature is what one would expect from strong interaction and QCD physics; if the length of confinement of a fermion is small, the energy required to overcome its confining barrier is large, and vice versa. Roughly speaking, one has a sort of uncertainty relation $L \sim 1 / p$, with $p$ being the momentum which, for relativistic particles, is proportional to the energy. Anyhow, the questions of how the fermions are confined and how they get unconfined are not answered with our analysis.

Since we have completely determined the relevant dependence of $L_{c}^{(D)}$ and $T_{d}^{(D)}$ on $\lambda$, for any value of $\lambda\left(\geq \lambda_{c}^{(D)}\right)$, estimates of values of the confining length and the deconfining temperature require the specification of the mass of the fermions. Viewing the GN model as an effective model for strong interaction, a natural choice is to take the constituent quark mass, $\mathrm{m} \approx 350 \mathrm{MeV} \simeq 1.75 \mathrm{fm}^{-1}$ [61]. With such a choice, we obtain the limiting values of the confining length and the deconfining temperature presented in Table I.

We find that the range of variation of $L_{c}^{(D)}$ and $T_{d}^{(D)}$, as $\lambda$ runs in the strong-coupling regime $\left(\geq \lambda_{c}^{(D)}\right)$, is relatively small and decreases as $D$ increases. These values compare amazingly well with the size of hadrons (e.g., the experimentally measured proton charge diameter is $\approx 1.74 \mathrm{fm}[62])$ and the estimated deconfining temperature $(\approx 200 \mathrm{MeV})$ for hadronic matter [63].

To summarize, we have established the existence of a phase transition in the massive large- $N$ GN model with compactified spatial dimensions. Further investigation is needed for other aspects of this transition like effects of finite density and critical exponents. Such a study is left for a future work.

\section{ACKNOWLEDGMENTS}

This work was partially supported by CNPq and CAPES (Brazil), and NSERC (Canada). 
[1] D. J. Gross, R. D. Pisarski, and L. G. Yaffe, Rev. Mod. Phys. 53, 43 (1981).

[2] O. K. Kalashnikov, Fortschr. Phys. 32, 525 (1984).

[3] Y. Nambu and G. Jona-Lasinio, Phys. Rev. 122, 345 (1961); 124, 246 (1961).

[4] D. J. Gross and A. Neveu, Phys. Rev. D 10, 3235 (1974).

[5] C. de Calan, P. A. Faria da Veiga, J. Magnen, and R. Séneor, Phys. Rev. Lett. 66, 3233 (1991).

[6] G. Parisi, Nucl. Phys. B100, 368 (1975).

[7] Y. Aref'eva, E. Nissimov, and S. Pacheva, Commun. Math. Phys. 71, 213 (1980).

[8] K. Gawedzki and A. Kupiainen, Phys. Rev. Lett. 55, 363 (1985); Nucl. Phys. B262, 33 (1985).

[9] B. Rosenstein, B. J. Warr, and S. H. Park, Phys. Rev. Lett. 62, 1433 (1989).

[10] S. Weinberg, Phys. Rev. D 56, 2303 (1997).

[11] U. Wolff, Phys. Lett. 157B, 303 (1985).

[12] A. Barducci, R. Casalbuoni, M. Modugno, G. Pettini, and R. Gatto, Phys. Rev. D 51, 3042 (1995).

[13] H. R. Christiansen, A. C. Petkou, M. B. Silva Neto, and N. D. Vlachos, Phys. Rev. D 62, 025018 (2000).

[14] S. J. Hands, J. B. Kogut, and C. G. Strouthos, Phys. Lett. B 515, 407 (2001).

[15] V. Juričić, I. F. Herbut, and G. W. Semenoff, Phys. Rev. B 80, 081405 (2009).

[16] B. R. Zhou, Phys. Rev. D 57, 3171 (1998); Commun. Theor. Phys. 32, 425 (1999).

[17] A. Brzoska and M. Thies, Phys. Rev. D 65, 125001 (2002).

[18] J.-L. Kneur, M. B. Pinto, R. O. Ramos, and E. Staudt, Phys. Rev. D 76, 045020 (2007).

[19] C. Boehmer, U. Fritsch, S. Kraus, and M. Thies, Phys. Rev. D 78, 065043 (2008).

[20] J. Feinberg, Int. J. Mod. Phys. A 17, 898 (2002).

[21] M. Thies, J. Phys. A 39, 12707 (2006).

[22] O. Schnetz, M. Thies, and K. Urlichs, Ann. Phys. (N.Y.) 321, 2604 (2006).

[23] F. Höfling, C. Nowak, and C. Wetterich, Phys. Rev. B 66, 205111 (2002).

[24] S. J. Hands, J. B. Kogut, and C. G. Strouthos, Phys. Rev. D 65, 114507 (2002).

[25] H. Kohyama, Phys. Rev. D 77, 045016 (2008).

[26] S. Doniach and E. H. Sondheimer, Green's Function for Solid State Physicists (Benjamin, New York, 1974).

[27] S. Weinberg, Prog. Theor. Phys. Suppl. 86, 43 (1986).

[28] F. C. Khanna, A. P. C. Malbouisson, J. M. C. Malbouisson, and A. E. Santana, Europhys. Lett. 92, 11001 (2010).

[29] N. D. Birrell and L. H. Ford, Phys. Rev. D 22, 330 (1980).

[30] J. Polchinski, Commun. Math. Phys. 104, 37 (1986).

[31] J. J. Atick and E. Witten, Nucl. Phys. B310, 291 (1988).

[32] F. C. Khanna, A. P. C. Malbouisson, J. M. C. Malbouisson, and A. E. Santana, Ann. Phys. (N.Y.) 324, 1931 (2009).

[33] F. C. Khanna, A. P. C. Malbouisson, J. M. C. Malbouisson, and A.E. Santana, Thermal Quantum Field Theory: Algebraic Aspects and Applications (World Scientific, Singapore, 2009).

[34] F. C. Khanna, A. P. C. Malbouisson, J. M. C. Malbouisson, and A. E. Santana, Ann. Phys. (N.Y.) 326, 2634 (2011).
[35] A. P. C. Malbouisson, J. M. C. Malbouisson, A. E. Santana, and J. C. Silva, Phys. Lett. B 583, 373 (2004).

[36] F. C. Khanna, A. P. C. Malbouisson, J. M. C. Malbouisson, H. Queiroz, T. M. Rocha-Filho, A. E. Santana, and J.C. Silva, Phys. Lett. B 624, 316 (2005).

[37] L. H. Ford and N. F. Svaiter, Phys. Rev. D 51, 6981 (1995).

[38] A. P. C. Malbouisson and J. M. C. Malbouisson, J. Phys. A 35, 2263 (2002).

[39] A.P.C. Malbouisson, J.M.C. Malbouisson, and A.E. Santana, Nucl. Phys. B631, 83 (2002).

[40] A. P. C. Malbouisson, J. M. C. Malbouisson, A. E. Santana, and F. C. Khanna, Mod. Phys. Lett. A 20, 965 (2005).

[41] L. M. Abreu, C. de Calan, A. P. C. Malbouisson, J. M. C. Malbouisson, and A. E. Santana, J. Math. Phys. (N.Y.) 46, 012304 (2005).

[42] A. P. C. Malbouisson, J.M.C. Malbouisson, and R. C. Pereira, J. Math. Phys. (N.Y.) 50, 083304 (2009).

[43] L. H. Ford, Phys. Rev. D 21, 933 (1980).

[44] H. Kleinert and A. Zhuk, Theor. Math. Phys. 108, 1236 (1996).

[45] J.C. da Silva, F. C. Khanna, A. Matos Neto, and A.E. Santana, Phys. Rev. A 66, 052101 (2002).

[46] H. Queiroz, J.C. da Silva, F.C. Khanna, J. M. C. Malbouisson, M. Revzen, and A. E. Santana, Ann. Phys. (N.Y.) 317, 220 (2005); 321, 1274 (2006).

[47] N. Ahmadi and M. Nouri-Zonoz, Phys. Rev. D 71, 104012 (2005).

[48] J. L. Tomazelli and L. C. Costa, Int. J. Theor. Phys. 45, 499 (2006).

[49] O. Kiriyama and A. Hosaka, Phys. Rev. D 67, 085010 (2003).

[50] L. M. Abreu, M. Gomes, and A. J. da Silva, Phys. Lett. B 642, 551 (2006).

[51] D. Ebert, K. G. Klimenko, A. V. Tyukov, and V.C. Zhukovsky, Phys. Rev. D 78, 045008 (2008).

[52] L. M. Abreu, A. P. C. Malbouisson, J. M. C. Malbouisson, and A. E. Santana, Nucl. Phys. 819, 127 (2009).

[53] L. M. Abreu, A.P.C. Malbouisson, and J.M.C. Malbouisson, Europhys. Lett. 90, 11001 (2010); Phys. Rev. D 83, 025001 (2011); 84, 065036 (2011).

[54] C. C. Ttira, C.D. Fosco, A.P.C. Malbouisson, and I. Roditi, Phys. Rev. D 77, 105030 (2008).

[55] A. Chodos, R. L. Jaffe, K. Johnson, C. B. Thorn, and V. F. Weisskopf, Phys. Rev. D 9, 3471 (1974).

[56] C. A. Lutken and F. Ravndal, J. Phys. A 21, L793 (1988).

[57] E. Elizalde and A. Romeo, J. Math. Phys. (N.Y.) 30, 1133 (1989).

[58] K. Kirsten, J. Math. Phys. (N.Y.) 35, 459 (1994).

[59] J. M. C. Malbouisson, F. C. Khanna, A. P. C. Malbouisson, and A. E. Santana, AIP Conf. Proc. 917, 170 (2007).

[60] A. P. C. Malbouisson, F. C. Khanna, J. M. C. Malbouisson, and A. E. Santana, Braz. J. Phys. 36, 1165 (2006). Some corrections to the numerical results of Refs. [35,36] have been presented in this reference.

[61] S. Eidelman et al. (Particle Data Group), Phys. Lett. B 592, 1 (2004); see p. 475.

[62] S. G. Karshenboim, Can. J. Phys. 77, 241 (1999).

[63] A. Smilga, Lectures on QCD (World Scientific, Singapore, 2001), p. 279. 